\title{
Cause and effects of the massive star formation in Messier 8 East
}

\author{
M. Tiwari ${ }^{1,2, \star}$, K. M. Menten ${ }^{1}$, F. Wyrowski ${ }^{1}$, A. Giannetti ${ }^{3}$, M.-Y. Lee ${ }^{4}$, W.-J. Kim ${ }^{5}$, and J. P. Pérez-Beaupuits ${ }^{6}$ \\ ${ }^{1}$ Max-Planck Institute for Radio Astronomy, Auf dem Hügel, 53121 Bonn, Germany \\ e-mail: mtiwari@mpifr-bonn.mpg.de \\ 2 Department of Astronomy, University of Maryland, College Park, MD 20742-2421, USA \\ 3 INAF-Istituto di Radioastronomia, and Italian ALMA Regional Centre, Via P. Gobetti 101, 40129 Bologna, Italy \\ ${ }^{4}$ Korea Astronomy and Space Science Institute Daedeokdae-ro 776, Yuseong-gu Daejeon 34055, Republic of Korea \\ ${ }^{5}$ Instituto de Radioastronomía Milimétrica, Avenida Divina Pastora 7, 18012 Granada, Spain \\ ${ }^{6}$ European Southern Observatory, Alonso de Córdova 3107,Vitacura Casilla 7630355, Santiago, Chile
}

Received 9 July 2020 / Accepted 16 October 2020

\begin{abstract}
Context. Messier 8 (M8), one of the brightest H II regions in our Galaxy, is powered by massive O-type stars and is associated with recent and ongoing massive star formation. Two prominent massive star-forming regions associated with M8 are M8-Main, the particularly bright part of the large-scale H II region (mainly) ionized by the stellar system Herschel 36 (Her 36) and M8 East (M8 E), which is mainly powered by a deeply embedded young stellar object (YSO), the bright infrared (IR) source M8E-IR.

Aims. We study the interaction of the massive star-forming region M8 E with its surroundings using observations of assorted diffuse and dense gas tracers that allow quantifying the kinetic temperatures and volume densities in this region. With a multiwavelength view of M8 E, we investigate the cause of star formation. Moreover, we compare the star-forming environments of M8-Main and M8 E, based on their physical conditions and the abundances of the various observed species toward them.

Methods. We used the Institut de Radioastronomía Millimétrica $30 \mathrm{~m}$ telescope to perform an imaging spectroscopy survey of the $\sim 1 \mathrm{pc}$ scale molecular environment of M8E-IR and also performed deep integrations toward the source itself. We imaged and analyzed data for the $J=1 \rightarrow 0$ rotational transitions of ${ }^{12} \mathrm{CO},{ }^{13} \mathrm{CO}, \mathrm{N}_{2} \mathrm{H}^{+}, \mathrm{HCN}, \mathrm{H}^{13} \mathrm{CN}, \mathrm{HCO}^{+}, \mathrm{H}^{13} \mathrm{CO}^{+}, \mathrm{HNC}$, and $\mathrm{HN}^{13} \mathrm{C}$ observed for the first time toward M8 E. To visualize the distribution of the dense and diffuse gas in M8 E, we compared our velocity-integrated intensity maps of ${ }^{12} \mathrm{CO},{ }^{13} \mathrm{CO}$, and $\mathrm{N}_{2} \mathrm{H}^{+}$with ancillary data taken at IR and submillimeter wavelengths. We used techniques that assume local thermodynamic equilibrium (LTE) and non-LTE to determine column densities of the observed species and constrain the physical conditions of the gas that causes their emission. Examining the class 0/ I and class II YSO populations in M8 E, allows us to explore the observed ionization front (IF) as seen in the high resolution Galactic Legacy Infrared Mid-Plane Survey Extraordinaire (GLIMPSE) $8 \mu \mathrm{m}$ emission image. The difference between the ages of the YSOs and their distribution in M8 E were used to estimate the speed of the IF.

Results. We find that ${ }^{12} \mathrm{CO}$ probes the warm diffuse gas also traced by the GLIMPSE $8 \mu \mathrm{m}$ emission, while $\mathrm{N}_{2} \mathrm{H}^{+}$traces the cool and dense gas following the emission distribution of the APEX Telescope Large Area Survey of the Galaxy $870 \mu \mathrm{m}$ dust continuum. We find that the star-formation in M8 E appears to be triggered by the earlier formed stellar cluster NGC 6530, which powers an $\mathrm{H}$ II region giving rise to an IF that is moving at a speed $\geq 0.26 \mathrm{~km} \mathrm{~s}^{-1}$ across M8 E. Based on our qualitative and quantitative analysis, the $J=1 \rightarrow 0$ transition lines of $\mathrm{N}_{2} \mathrm{H}^{+}$and $\mathrm{HN}^{13} \mathrm{C}$ appear to be more direct tracers of dense molecular gas than the $J=1 \rightarrow 0$ transition lines of $\mathrm{HCN}$ and $\mathrm{HCO}^{+}$. We derive temperatures of 80 and $30 \mathrm{~K}$ for the warm and cool gas components, respectively, and constrain the $\mathrm{H}_{2}$ volume densities to be in the range of $10^{4}-10^{6} \mathrm{~cm}^{-3}$. Comparison of the observed abundances of various species reflects the fact that M8 $\mathrm{E}$ is at an earlier stage of massive star formation than M8-Main.
\end{abstract}

Key words. ISM: abundances - ISM: molecules - submillimeter: ISM - stars: formation - ISM: individual objects: M 8

\section{Introduction}

Massive stars contribute significantly to the evolution of galaxies by injecting radiative and mechanical energy into the interstellar medium (ISM). This energy input stirs the environment around massive stars through stellar winds, ionization, and heating of the gas, and through supernovae explosions. All these processes considerably change the chemical composition and structure of the ISM in their neighborhood (for overviews, see Tielens 2010, 2013; Draine 2011). The interaction of massive stars with their surroundings can thus affect the star formation process either by quenching star formation or by triggering it: formation of

\footnotetext{
* Member of the International Max Planck Research School (IMPRS) for Astronomy and Astrophysics at the Universities of Bonn and Cologne.
}

cloud and intercloud phases in the ISM, leading to disruption of molecular clouds, can halt star formation, while compression of the surrounding gas or the propagation of photoionizationinduced shocks can set off star formation, a scenario originating with Elmegreen \& Lada (1977); see, for instance, Urquhart et al. (2007) and Kim et al. (2013) for recent observational studies. Massive stars give rise to bright $\mathrm{H}$ II regions and photodissociation regions (PDRs). H II regions comprise hot ionized gas irradiated by strong UV $(\mathrm{h} v>13.6 \mathrm{eV})$ radiation from one or more nearby hot luminous stars. PDRs are at the interface of these H II regions and the cool molecular cloud shielded from UV radiation from the illuminating star (Hollenbach \& Tielens 1999). In PDRs, the thermal and chemical processes are regulated by far-UV (FUV, $6 \mathrm{eV}<\mathrm{h} v<13.6 \mathrm{eV}$ ) photons. In order to understand how the ISM is affected by the interaction with 


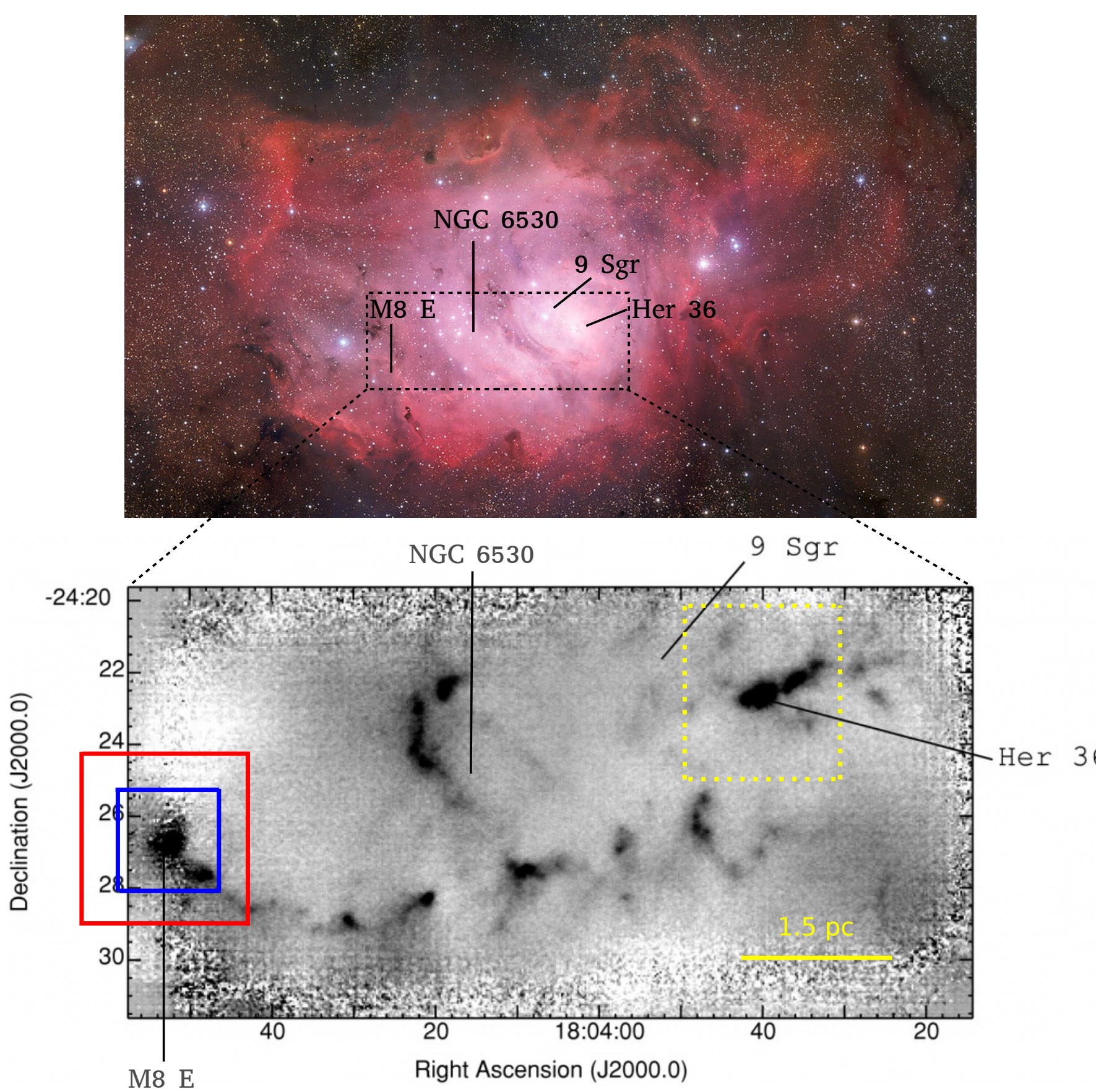

Fig. 1. Upper panel: image of M8 taken with the WFI attached to the MPG/ESO $2.2 \mathrm{~m}$ telescope at the La Silla Observatory. The image is based on the maps observed with three different broadband filters: $B$ (at $\sim 456.252 \mathrm{~nm}$ ), $V$ (at $539.562 \mathrm{~nm}$ ), and $R$ (at $651.725 \mathrm{~nm}$ ) and one narrow-band filter: $H \alpha$ (at $658.827 \mathrm{~nm})$. Lower panel: map of M8 at $450 \mu \mathrm{m}$ adopted from Tothill et al. (2002, Fig. 2). The boundaries of the observed maps shown in Fig. 3 are marked in red (Fig. 3, top row) and blue (Fig. 3, middle and bottom rows). Our observed maps extend farther to the east, beyond the edge of this image. The boundary of the observed maps of various species reported in Tiwari et al. (2018) is shown as yellow dots. The positions of the luminous stellar systems Her 36 and 9 Sgr, which are located in the central optically bright region of M8, are marked in both panels, along with the position of the open cluster NGC 6530 and the eastern star-forming region M8-E.

massive stars, it is of fundamental importance to study massive star-forming regions.

Messier 8 (M8), the Lagoon nebula, is one of the most prominent $\mathrm{H}$ II regions in our galaxy. Associated with is central region is a well-developed PDR (Tiwari et al. 2018) that represents the second most intense ${ }^{12} \mathrm{CO}$ emission source known (in 1996) (White et al. 1997). Located in the Sagittarius-Carina arm, near our line of sight toward the Galactic center, it is relatively close to the Sun, at a distance of $\sim 1.25 \mathrm{kpc}$ ( $1^{\prime}$ corresponding to
0.36 pc) (Damiani et al. 2004 and Arias et al. 2006). Recently, this distance has been confirmed by Gaia parallaxes that yield a value of $1.35 \mathrm{kpc}$ with an uncertainty of 9\% (Damiani et al. 2019). M8 (Fig. 1) as a whole is an extended H II region ( $\sim 10 \mathrm{pc}$ across) that is powered by the open stellar cluster NGC 6530 (Prisinzano et al. 2005), which contains several O-type stars, and by the spectroscopic binary 9 Sagittarii $(9 \mathrm{Sgr})$ consisting of an O3.5 V and an O5-5.5 V star (Rauw et al. 2012). M8 is also associated with two major regions with more recent star formation 
Table 1. Molecular lines mapped in the M8 E region.

\begin{tabular}{|c|c|c|c|c|c|c|c|c|}
\hline Species/Line & $\begin{array}{c}v \\
(\mathrm{GHz})\end{array}$ & $\begin{array}{l}E_{\text {up }} \\
(\mathrm{K})\end{array}$ & $\begin{array}{c}\theta \\
\left({ }^{\prime \prime}\right)\end{array}$ & $\begin{array}{c}\int T_{\mathrm{MB}} \mathrm{d} v(\text { peak }) \\
\left(\mathrm{K} \mathrm{km} \mathrm{s}^{-1}\right)\end{array}$ & $\begin{array}{c}v_{\mathrm{LSR}} \\
\left(\mathrm{km} \mathrm{s}^{-1}\right)\end{array}$ & $\begin{array}{c}\mathrm{rms} \\
\left(\mathrm{K} \mathrm{km} \mathrm{s}^{-1}\right)\end{array}$ & $\begin{array}{l}n_{\text {crit }}{ }^{(a)} \\
\left(\mathrm{cm}^{-3}\right)\end{array}$ & $\begin{array}{c}\text { Size } \\
\left({ }^{\prime \prime} \times{ }^{\prime \prime}\right)\end{array}$ \\
\hline${ }^{12} \mathrm{CO}(1 \rightarrow 0)$ & 115.2712 & 5.5 & 22.5 & 440.2 & 10.5 & 0.71 & $2.2 \times 10^{3}$ & $260 \times 260$ \\
\hline${ }^{13} \mathrm{CO}(1 \rightarrow 0)$ & 110.2013 & 5.3 & 23.5 & 86.9 & 9.9 & 0.33 & $1.9 \times 10^{3}$ & $260 \times 260$ \\
\hline $\mathrm{N}_{2} \mathrm{H}^{+}(1 \rightarrow 0)$ & 93.1733 & 4.5 & 27.8 & 20.3 & 10.7 & 0.21 & $3.7 \times 10^{5}$ & $260 \times 260$ \\
\hline $\mathrm{HCN}(1 \rightarrow 0)$ & 88.6316 & 4.3 & 29.3 & 77.7 & 10.6 & 0.17 & $1 \times 10^{6}$ & $160 \times 160$ \\
\hline $\mathrm{H}^{13} \mathrm{CN}(1 \rightarrow 0)$ & 86.3399 & 4.1 & 30.0 & 9.0 & 10.8 & 0.12 & & $160 \times 160$ \\
\hline $\mathrm{HCO}^{+}(1 \rightarrow 0)$ & 89.1885 & 4.3 & 29.1 & 42.6 & 10.8 & 0.21 & $1.9 \times 10^{5}$ & $160 \times 160$ \\
\hline $\mathrm{H}^{13} \mathrm{CO}^{+}(1 \rightarrow 0)$ & 86.7542 & 4.2 & 30.0 & 3.8 & 10.7 & 0.16 & $1.8 \times 10^{5}$ & $160 \times 160$ \\
\hline $\mathrm{HNC}(1 \rightarrow 0)$ & 90.6635 & 4.4 & 28.6 & 25.4 & 10.8 & 0.21 & $3.2 \times 10^{5}$ & $160 \times 160$ \\
\hline $\mathrm{HN}^{13} \mathrm{C}(1 \rightarrow 0)$ & 87.0908 & 4.2 & 30.0 & 1.9 & 10.8 & 0.17 & & $160 \times 160$ \\
\hline
\end{tabular}

Notes. Columns are from left to right the species/line, frequency, upper level energy, FWHM beam size, peak-integrated flux density in the map, centroid LSR velocity, rms noise, critical density for optically thin emission, and map size. The measured line parameters (flux, centroid LSR velocity, and rms) are for the central position, which corresponds to that of M8E-IR and is given in Sect. 2. ${ }^{(a)}$ Critical densities calculated for species whose collisional rate coefficients are provided by LAMDA. For ${ }^{12} \mathrm{CO}$ and ${ }^{13} \mathrm{CO}$, the coefficients are determined at $80 \mathrm{~K}$ and for $\mathrm{N}_{2} \mathrm{H}^{+}$, $\mathrm{HCN}, \mathrm{HCO}^{+}, \mathrm{H}^{13} \mathrm{CO}^{+}$, and $\mathrm{HNC}$, the coefficients are determined at $30 \mathrm{~K}$.

activity: one near its brightest part, the Hourglass nebula (hereafter M8-Main) that is illuminated by the bright and very young multiple stellar system Herschel 36 (Her 36) (O7.5 V + O9 V + B0.5 V, Arias et al. 2010) and a region to the southeast, M8 east (M8 E), where at least two massive stars have very recently formed, and whose dust continuum emission rivals in brightness that of M8-Main at far-IR (FIR) and submillimeter wavelengths (Tothill et al. 2008).

Using the Stratospheric Observatory for Infrared Astronomy (SOFIA, Young et al. 2012), the Atacama Pathfinder EXperiment 12 m submilimeter telescope (APEX, Güsten et al. 2006), and the $30 \mathrm{~m}$ millimeter telescope operated by the Institut de Radioastronomía Millimétrica (IRAM ${ }^{1}$ ) on Pico Veleta in Spain, we performed an extensive survey of M8-Main, as reported in two recent publications: We described the morphology of the volume around Her 36 and determined the physical conditions of the gas surrounding it (Tiwari et al. 2018) and shed some light on the formation process of hydrocarbons in M8-Main, which is a high UV flux PDR with $G_{0} \sim 10^{5}$ in Habing units (Tiwari et al. 2019).

$\mathrm{M} 8 \mathrm{E}$ is a massive star-forming region about $5 \mathrm{pc}$ in projected distance away from M8-Main and it lies within a molecular cloud with a size of a few arcmin (roughly 1 pc) (Tothill et al. 2008). A small $\left(<0.13 \mathrm{pc}^{2} \sim 1 \operatorname{arcmin}^{2}\right)$ region contains a quite rich embedded cluster comprising 7 IR sources that is associated with the molecular gas. It includes a zero age main-sequence (ZAMS) B2 star, powering a very small $\left(0 .{ }^{\prime \prime} 6 \sim 750 \mathrm{AU}\right.$ in diameter $)$ ultracompact $\mathrm{H}$ II region, known as M8E-Radio, and only $\sim 7^{\prime \prime}$ $(0.04 \mathrm{pc})$ away, a massive young stellar object (YSO), M8E-IR, which is likely to become a B0 star and dominates the near- and mid-IR radiation of the cluster up to a wavelength of $\sim 24 \mu \mathrm{m}$ (Linz et al. 2008). In the past, (sub)mm wavelength imaging of M8 $\mathrm{E}$ has targeted dust continuum emission at 1300, 850 and $450 \mu \mathrm{m}$ and resulted in the detection of a number of cores and also CO emission (Tothill et al. 2002; Zhang et al. 2005). In particular, high-velocity molecular gas tracing a molecular outflow was discovered to originate in M8E-IR by mapping the ${ }^{12} \mathrm{CO} J=2 \rightarrow 1$ transition (Mitchell et al. 1992; Zhang et al. 2005).

1 IRAM is supported by INSU/CNRS, the MPG (Germany), and IGN (Spain).
Quite surprisingly, the literature on observations of M8 E in lines from molecules other than $\mathrm{CO}$ is quite sparse. The goal of this study was to collect and analyze data from a variety of molecular lines to establish an inventory of the observed diffuse and dense gas tracers that can be used to investigate the physical and chemical conditions in the M8 E massive starforming region and compare this molecular environment with that of M8-Main. Our observations were done in the $3 \mathrm{~mm}$ band using the Eight MIxer Receiver (EMIR ${ }^{2}$, Carter et al. 2012) on the IRAM $30 \mathrm{~m}$ telescope. In this paper, we present observations of various diffuse and dense gas tracers found toward M8 E that consisted of a mapping survey with different frequency coverage of a large $\left(260^{\prime \prime} \times 260^{\prime \prime}\right)$ and a smaller $\left(160^{\prime \prime} \times 160^{\prime \prime}\right)$ region $(\approx 1.6 \mathrm{pc} \times 1.6 \mathrm{pc}$ and $1 \mathrm{pc} \times 1 \mathrm{pc}$, respectively) centered on M8E-IR. The observations are described in Sect. 2, and velocity-integrated intensity maps of the various observed species are presented in Sect. 3. The data are analyzed quantitatively in Sect. 4. Finally, we discuss the results and summarize the main conclusions of this work in Sects. 5 and 6 and give a brief outlook on further studies of the interesting environment of M8 in Sect. 7.

\section{Observations}

We used the IRAM 30 m telescope in 2016 August for observations of M8 E covering most of the $3 \mathrm{~mm}$ atmospheric window. We employed EMIR as a frontend, followed by a fast Fourier transform spectrometer (FFTS) as a backend. The FFTS channel spacing of $200 \mathrm{kHz}$ corresponds to a velocity resolutions of 0.70 and $0.52 \mathrm{~km} \mathrm{~s}^{-1}$ at the frequencies of the lowest and highest frequency lines under discussion here, 85.4 and $115.3 \mathrm{GHz}$, respectively. We focus on the $J=1 \rightarrow 0$ transitions of ${ }^{12} \mathrm{CO}$, ${ }^{13} \mathrm{CO}, \mathrm{N}_{2} \mathrm{H}^{+}, \mathrm{HCN}, \mathrm{H}^{13} \mathrm{CN}, \mathrm{HCO}^{+}, \mathrm{H}^{13} \mathrm{CO}^{+}, \mathrm{HNC}$, and $\mathrm{HN}^{13} \mathrm{C}$, and on the $J=5 \rightarrow 4$ and $J=6 \rightarrow 5$ multiple $K$ series of $\mathrm{CH}_{3} \mathrm{CCH}$. Information on the observed $J=1 \rightarrow 0$ lines is given in Table 1 , while Table 2 summarizes the $\mathrm{CH}_{3} \mathrm{CCH}$ lines. The full molecular inventory of M8 $\mathrm{E}$ will be discussed in a forthcoming publication.

\footnotetext{
2 http://WWW.iram.es/IRAMES/mainWiki/ EmirforAstronomers
} 
Table 2. Observed spectral line parameters of $\mathrm{CH}_{3} \mathrm{CCH}$.

\begin{tabular}{ccccc}
\hline \hline Transition & Frequency $(\mathrm{GHz})$ & $E_{\text {up }}(\mathrm{K})$ & $T_{\text {peak }}(\mathrm{K})$ & $\mathrm{rms}(\mathrm{K})$ \\
\hline$J, K=5,4 \rightarrow 4,4$ & 85.4312 & 127.9 & $<3 \times \mathrm{rms}$ & \\
$J, K=5,3 \rightarrow 4,3$ & 85.4425 & 77.3 & 0.137 & \\
$J, K=5,2 \rightarrow 4,2$ & 85.4507 & 41.2 & 0.204 & 0.026 \\
$J, K=5,1 \rightarrow 4,1$ & 85.4556 & 19.5 & 0.464 & \\
$J, K=5,0 \rightarrow 4,0$ & 85.4572 & 12.3 & 0.630 & \\
\hline$J, K=6,5 \rightarrow 5,5$ & 102.4991 & 197.8 & $<3 \times \mathrm{rms}$ & \\
$J, K=6,4 \rightarrow 5,4$ & 102.5165 & 132.8 & $<3 \times \mathrm{rms}$ & \\
$J, K=6,3 \rightarrow 5,3$ & 102.5303 & 82.3 & 0.162 & 0.030 \\
$J, K=6,2 \rightarrow 5,2$ & 102.5401 & 46.1 & 0.298 & \\
$J, K=6,1 \rightarrow 5,1$ & 102.5460 & 24.5 & 0.696 & \\
$J, K=6,0 \rightarrow 5,0$ & 102.5479 & 17.2 & 0.796 & \\
\hline
\end{tabular}

Mapping observations in on-the-fly (OTF) total power mode were centered on M8E-IR at right ascension and declination, $(\alpha, \delta)_{\mathrm{J} 2000}=18^{\mathrm{h}} 04^{\mathrm{m}} 53 . .^{\mathrm{s}} 3$, and $-24^{\circ} 26^{\prime} 42 .^{\prime \prime} 3$, respectively (Tothill et al. 2008). The ${ }^{12} \mathrm{CO},{ }^{13} \mathrm{CO}$, and $\mathrm{N}_{2} \mathrm{H}^{+}$line emission maps have a size of $\sim 260^{\prime \prime} \times 260^{\prime \prime}$, and the $\mathrm{HCN}, \mathrm{H}^{13} \mathrm{CN}, \mathrm{HCO}^{+}$, $\mathrm{H}^{13} \mathrm{CO}^{+}, \mathrm{HNC}$, and $\mathrm{HN}^{13} \mathrm{C}$ line maps have a size of $\sim 160^{\prime \prime} \times$ $160^{\prime \prime}$. Each subscan lasted $25 \mathrm{~s}$, and the integration time on the off-source reference position was $5 \mathrm{~s}$. The offset position relative to the center was at $\left(30^{\prime},-30^{\prime}\right)$ and the pointing accuracy $\left(<3^{\prime \prime}\right)$ was maintained by pointing at the bright calibrator, the QSO B1757-240, every 1-1.5 h. We also performed pointed observations with deep integrations toward the M8E-IR position, which provided us with the better signal-to-noise ratio $(\mathrm{S} / \mathrm{N})$ required for our spectral analysis and from which the $\mathrm{CH}_{3} \mathrm{CCH}$ data profit. A forward-coupling efficiency, $\eta_{\mathrm{f}}$, of 0.94 and a mainbeam efficiency, $\eta_{\mathrm{MB}}$, of 0.7 were adopted for the $3 \mathrm{~mm}$ module of the EMIR receiver. These efficiencies are defined and their values reported on the IRAM $30 \mathrm{~m}$ efficiencies website ${ }^{3}$ and in the (2015) IRAM commissioning report that is also linked in the above mentioned website.

We reduced of the calibrated data to produce spectra and maps with the Continuum and Line Analysis Single dish Software (CLASS) and the GREnoble Graphic (GREG) software, which are a part of the Grenoble Image and Line Data Analysis Software $\left(\right.$ GILDAS $^{4}$, Pety 2005) package. A part of the data analysis was made using matplotlib (Hunter 2007), which is a python library. All observations are summarized in Tables 1 and 2.

\section{Results}

\subsection{Observed spectra toward M8E-IR}

Figure 2 shows the spectra of the species listed in Table 1, observed along the brightest line of sight (LOS), that is, toward M8E-IR. The ${ }^{12} \mathrm{CO}$ and ${ }^{13} \mathrm{CO}$ line parameters were derived from two-component Gaussian fits, where initial guesses of $5-20 \mathrm{~km} \mathrm{~s}^{-1}$ and $24-30 \mathrm{~km} \mathrm{~s}^{-1}$ were provided. For $\mathrm{HCO}^{+}$, $\mathrm{H}^{13} \mathrm{CO}^{+}, \mathrm{HNC}$, and $\mathrm{HN}^{13} \mathrm{C}$, the line parameters were derived from single-component Gaussian fits. The line parameters of $\mathrm{N}_{2} \mathrm{H}^{+}, \mathrm{HCN}$, and $\mathrm{H}^{13} \mathrm{CN}$ were obtained from fits that considered the hyperfine structure (hfs) of these molecules. The derived

\footnotetext{
3 http://WWW.iram.es/IRAMES/mainWiki/

Iram30mEfficiencies

4 wWW.iram.fr/IRAMFR/GILDAS/
}

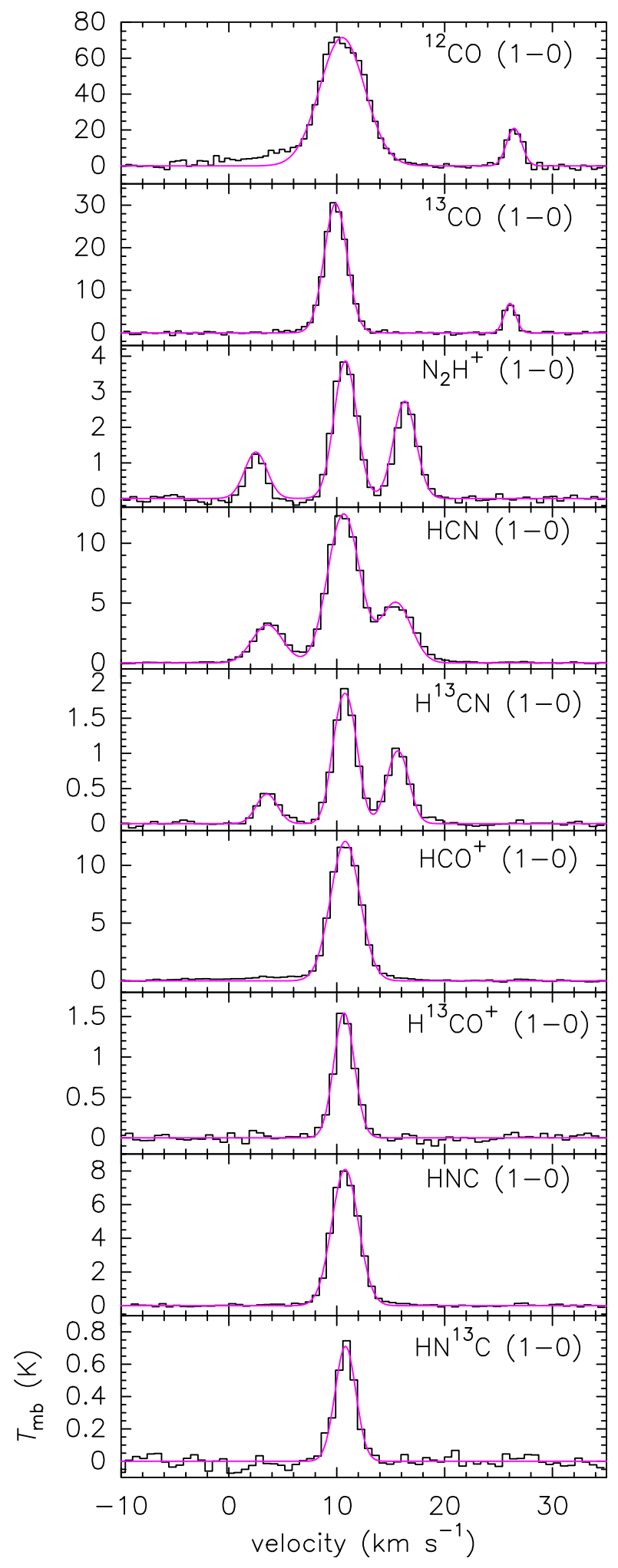

Fig. 2. Spectra of the species listed in Table 1, observed toward M8E-IR (black) with Gaussian fits (in pink), to a single line and, for the $\mathrm{N}_{2} \mathrm{H}^{+}$, $\mathrm{HCN}$, and $\mathrm{H}^{13} \mathrm{CN}$ lines to their hfs components.

centroid velocities are listed in Table 1 . For ${ }^{12} \mathrm{CO}$ and ${ }^{13} \mathrm{CO}$, the centroid velocity of the brightest component is mentioned. Similarly, for $\mathrm{N}_{2} \mathrm{H}^{+}, \mathrm{HCN}$, and $\mathrm{H}^{13} \mathrm{CN}$, the centroid velocity of the brightest hfs component is given.

The systemic LSR velocity of M8 E thus determined is $\approx+11 \mathrm{~km} \mathrm{~s}^{-1}$, which is similar to the +6 to $+11 \mathrm{~km} \mathrm{~s}^{-1}$ found for 
M8-Main (Tiwari et al. 2018). This similarity clearly indicates that both regions are in the same complex, although at different stages of their evolution. In addition, the ${ }^{12} \mathrm{CO}$ and ${ }^{13} \mathrm{CO}$ lines emit at a very different LSR velocity, $\sim+26 \mathrm{~km} \mathrm{~s}^{-1}$, which corresponds to a different molecular cloud along the LOS to M8 that is unrelated to this region (Dame et al. 2001). This other velocity component was also reported by Mitchell et al. (1992) in their ${ }^{12} \mathrm{CO} J=2 \rightarrow 1$ spectrum. Velocity-integrated intensity maps of the $J=1 \rightarrow 0$ transition of ${ }^{12} \mathrm{CO}$ and ${ }^{13} \mathrm{CO}$ from the $+26 \mathrm{~km} \mathrm{~s}^{-1}$ cloud are shown in Fig. A.1.

\subsection{Spatial distribution of the molecular line emission}

Table 1 lists, the line parameters determined from our spectra for all the observed molecular lines, which include the emission maxima and rms noise as obtained from the velocity-integrated intensity maps and the calculated critical densities (for optically thin emission). Figure 3 shows velocity-integrated intensity maps of the $J=1 \rightarrow 0$ transitions of ${ }^{12} \mathrm{CO},{ }^{13} \mathrm{CO}, \mathrm{N}_{2} \mathrm{H}^{+}, \mathrm{HCN}$, $\mathrm{H}^{13} \mathrm{CN}, \mathrm{HCO}^{+}, \mathrm{H}^{13} \mathrm{CO}^{+}, \mathrm{HNC}$, and $\mathrm{HN}^{13} \mathrm{C}$. The intensities of the $\mathrm{N}_{2} \mathrm{H}^{+}$and $\mathrm{HCN}$ lines were integrated over a velocity range of $0-20 \mathrm{~km} \mathrm{~s}^{-1}$ and -5 to $20 \mathrm{~km} \mathrm{~s}^{-1}$, respectively, in order to cover the hfs components of these lines. The emission intensities from all molecules peak at or very close to the position of M8E-IR. As expected, the distribution of the ${ }^{12} \mathrm{CO}$ emission is spread out the most, while the regions showing $\mathrm{H}^{13} \mathrm{CN}$ and $\mathrm{N}_{2} \mathrm{H}^{+}$ emission are the most concentrated. To quantify the extent of the emission regions, we determined their angular sizes by fitting 2D Gaussians to the velocity-integrated intensity maps, such that a contour plot at a cut through the Gaussian will be an ellipse. We used the "run gauss_2d" command in the GILDAS software for this. The elliptical emission size can be derived by defining boundaries of emission in our intensity maps and by guessing input values for the peak intensity and its corresponding position. The fit resulted in an angular size of the emitting region of $\mathrm{H}^{13} \mathrm{CN}$ of $\sim 40^{\prime \prime}$ and for that of $\mathrm{N}_{2} \mathrm{H}^{+}$of $\sim 72^{\prime \prime}$. The ${ }^{12} \mathrm{CO}$ and ${ }^{13} \mathrm{CO}$ emission extends over a larger area to the southwest and northeast of M8E-IR. Molecules with high critical densities $\left[\sim 10^{4}-10^{5} \mathrm{~cm}^{-3}\right.$ (Shirley 2015)] such as $\mathrm{HCN}, \mathrm{HCO}^{+}$, and $\mathrm{HNC}$ also show this extended emission, but more pronounced in the southwest direction than to the northeast. In contrast, only the southwest branch is seen in the lines of the isotopologues of these molecules, which have similar critical densities. In addition to the brightest emission toward M8E-IR, the intensity distribution of $\mathrm{N}_{2} \mathrm{H}^{+}$shows a secondary peak southwest of M8E-IR $\left(\Delta \alpha=-55^{\prime \prime}, \Delta \delta=-55^{\prime \prime}\right)$.

Because of their high spontaneous emission rates, which result in high critical densities, the $J=1 \rightarrow 0$ transition of the $\mathrm{HCN}, \mathrm{HCO}^{+}$, and $\mathrm{HNC}$ lines are generally considered to trace dense molecular gas. However, recent studies have shown that the $J=1 \rightarrow 0$ transition of $\mathrm{N}_{2} \mathrm{H}^{+}$is a more reliable dense gas tracer than the same transition of $\mathrm{HCN}, \mathrm{HCO}^{+}$, and $\mathrm{HNC}$ (Kauffmann et al. 2017, Pety et al. 2017, and Brinkmann et al. 2020). Despite having high critical densities, the $J=1 \rightarrow 0$ transitions of $\mathrm{HCN}, \mathrm{HCO}^{+}$, and $\mathrm{HNC}$ (and other species) were observed in the giant molecular clouds (GMCs) Orion A and B and W49 from relatively low-density gas $\left(n_{\mathrm{H}} \sim 10^{3} \mathrm{~cm}^{-3}\right)$; thus the gas that emits these species is not necessarily cool or dense (Kauffmann et al. 2017, Pety et al. 2017, and Barnes et al. 2020). Moreover, Goldsmith \& Kauffmann (2017) reported that if the electron abundance is about $X\left(\mathrm{e}^{-}\right)>10^{-5}$ or higher, HCN can be excited by collisions with electrons in regions in which the $\mathrm{H}_{2}$ densities are much lower than the mentioned critical density.
Moreover, the emission distribution of $\mathrm{HN}^{13} \mathrm{C}$ follows that of $\mathrm{N}_{2} \mathrm{H}^{+}$in $\mathrm{M} 8 \mathrm{E}$, similar to what was found for OMC-1 by Nakamura et al. (2019). Both $\mathrm{N}_{2} \mathrm{H}^{+}$and $\mathrm{HN}^{13} \mathrm{C}$ probe gas with very high visual extinction $A_{\mathrm{v}}>35 \mathrm{mag}$ (Pety et al. 2017), which means that they trace dust-embedded cores in molecular clouds.

The emission intensity of all molecules decreases dramatically to the north (from $\sim \Delta \delta=100^{\prime \prime}$, visible in ${ }^{12} \mathrm{CO}$ and ${ }^{13} \mathrm{CO}$ maps) and northwest (from $\sim \Delta \alpha=40^{\prime \prime}$, visible in all maps) of M8E-IR because M8 E is located at the edge of the compressed and warm molecular cloud that is strongly confined by the central H II region of M8 (Tothill et al. 2002). In order to visualize this steep edge, we plotted the emission distribution profiles of ${ }^{12} \mathrm{CO}, \mathrm{HCN}, \mathrm{HCO}^{+}$, and $\mathrm{N}_{2} \mathrm{H}^{+}$along two cuts, which are shown in Fig. 4. We compare the progression of the intensities of various species along these cuts in Fig. 5. Along cut (a), the emission of all species peaks at an offset $0^{\prime \prime}$ (corresponding to the position of M8E-IR) and decreases gradually toward the east and west of it. Along cut (b), however, the intensities suddenly drop away from M8E-IR to the northwest. The observed width of this edge, $\sim 17^{\prime \prime}$, is a little larger than the half-width $(0.5 \times$ the full width at half maximum, FWHM) of the $\mathrm{CO}$ beam as shown in Fig. 5b. This means that we are able to resolve this edge, but only slightly.

\subsection{Ancillary data}

To explore the relationship between the cool molecular cloud and the hot ionized gas, we compared our molecular line data with data from studies conducted at other wavelengths, mainly with the results of observations made in the IR and submm regimes. First, we extracted the $22 \mu \mathrm{m}$ dust continuum image from the all-sky Wide-Field Infrared Survey Explorer (WISE, Wright et al. 2010). Mid-IR dust continuum emission probes the warm dust in $\mathrm{H}$ II regions, which absorbs UV and FUV radiation from a nearby massive star and reemits in the MIR regime. The $22 \mu \mathrm{m}$ emission wavelength is similar to that of the Multiband Infrared Photometer for Spitzer on board the Spitzer Space Telescope (MIPSGAL, Carey et al. 2009), whose $24 \mu \mathrm{m}$ band data have been widely studied and found to be a direct tracer of $\mathrm{H}$ II regions, which correlates very well with the $21 \mathrm{~cm}$ radio continuum emission, because the hot plasma that gives rise to the free-free thermal emission at radio wavelengths is ionized by the same sources that cause the IR emission (Bania et al. 2010 and Anderson et al. 2014). Second, we used the $870 \mu \mathrm{m}$ dust continuum data from the APEX Telescope Large Area Survey of the Galaxy (ATLASGAL; Schuller et al. 2009), which is an unbiased survey covering 420 square degrees of the inner Galactic plane and was performed with the Large APEX BOlometer CAmera (LABOCA) instrument of the APEX 12 m telescope. Dust continuum emission in the submm range probes dense and cool clumps in the ISM and thus traces the early phases of (massive) star formation, including the earliest prestellar state. Figures $6 \mathrm{a}$ and b, show contours representing the $22 \mu \mathrm{m}$ and the $870 \mu \mathrm{m}$ emission overlaid on the velocity-integrated intensity map of $J=1 \rightarrow 0$ transition of ${ }^{13} \mathrm{CO}$, represented by the color scale in the background. Both the IR and submm continuum emission peak very close to M8E-IR (marked with an asterisk) and M8E-radio (marked with a triangle). While the $24 \mu \mathrm{m}$ emission probes the warm dust heated by the H II region, the $870 \mu \mathrm{m}$ ATLASGAL emission follows the structure of the ${ }^{13} \mathrm{CO}$ emission distribution better, with a bright extension toward the southwest of M8E-IR. To investigate further the nature of this bright southwest extension further, we overlaid the contours of the ATLASGAL $870 \mu \mathrm{m}$ emission on the velocity-integrated intensity map of the $\mathrm{N}_{2} \mathrm{H}^{+}$ $J=1 \rightarrow 0$ line (as the color-scale background). Figure 6c shows 


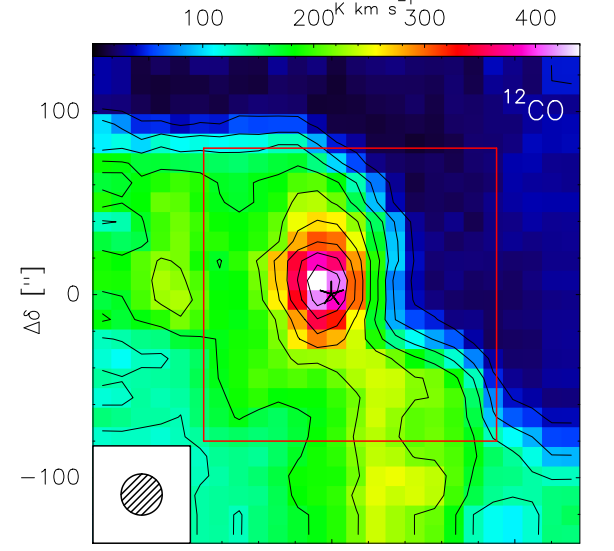

$\triangle \alpha$ ["]

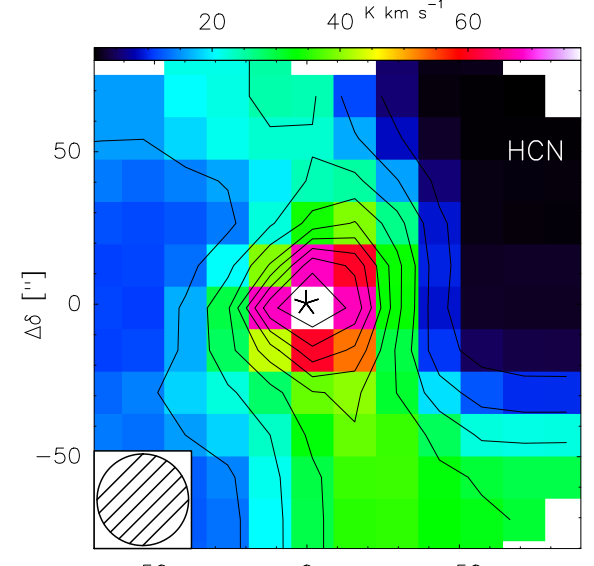

$\Delta \alpha["]$

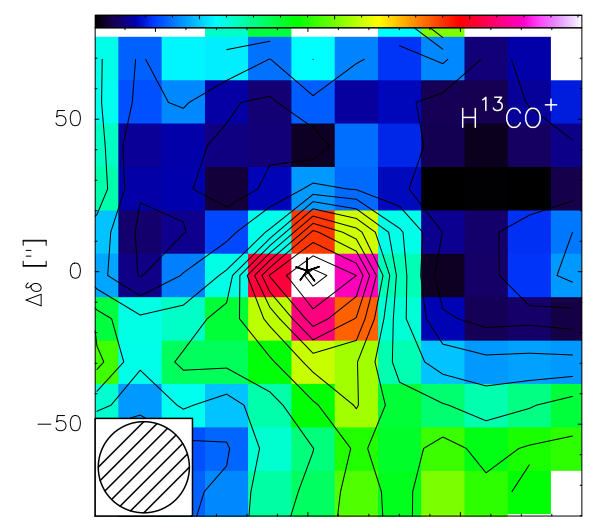

$\Delta \alpha$ ["]

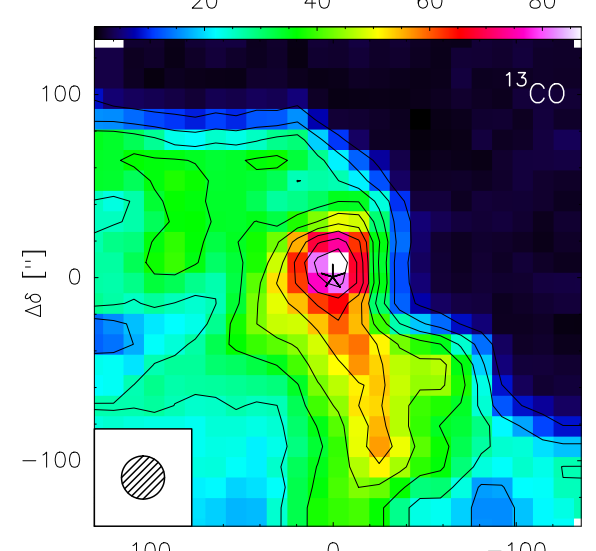

$\triangle a$ ["]

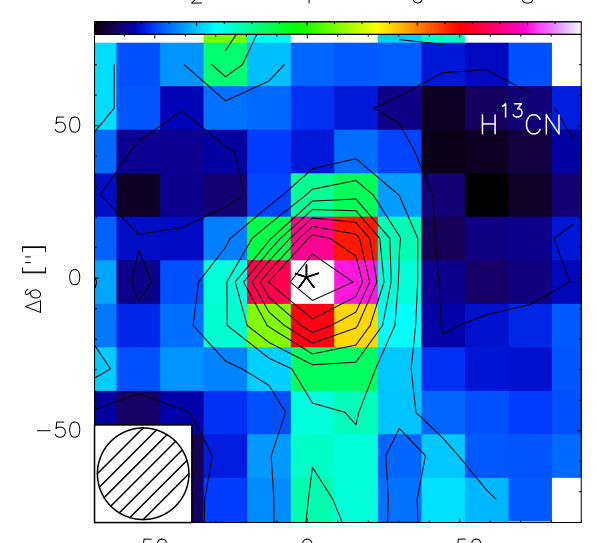

$\Delta \alpha$ ["]

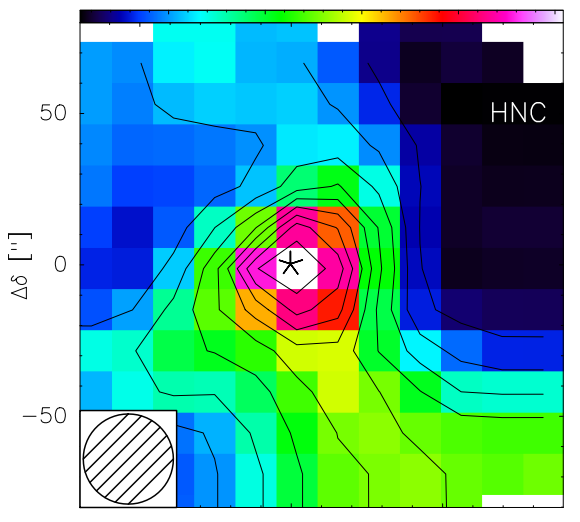

$\Delta \alpha\left[{ }^{\prime \prime}\right]$

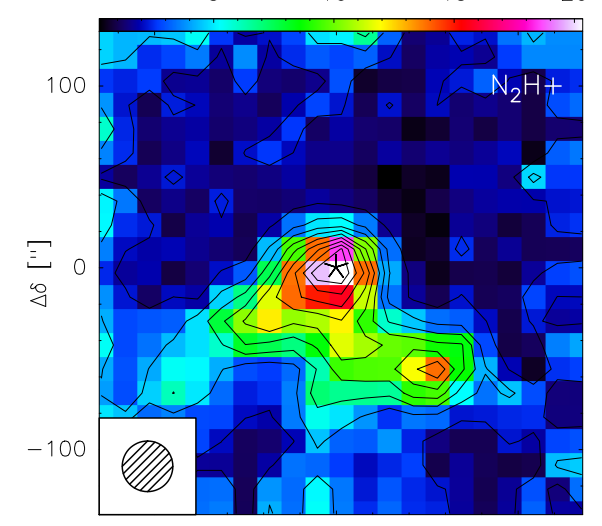

$\Delta \alpha["]$

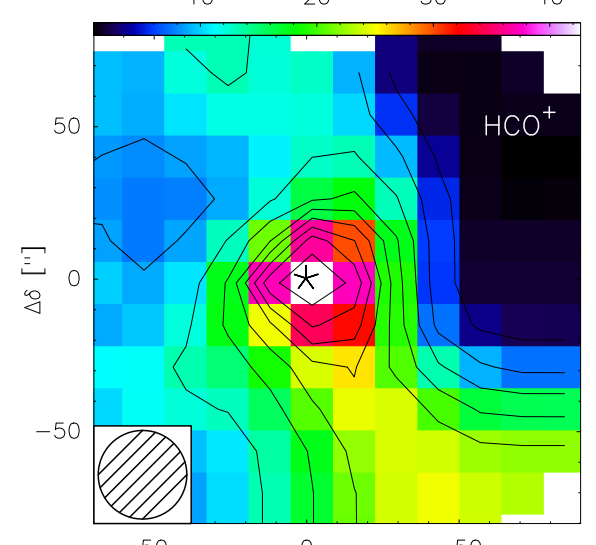

$\Delta \alpha["]$

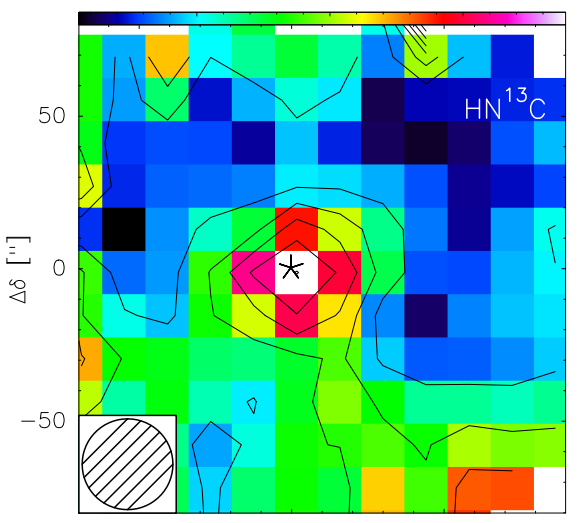

$\Delta \alpha["]$

Fig. 3. Color maps of the velocity-integrated intensity of the $J=1 \rightarrow 0$ transition of ${ }^{12} \mathrm{CO},{ }^{13} \mathrm{CO}, \mathrm{N}_{2} \mathrm{H}^{+}, \mathrm{HCN}, \mathrm{H}^{13} \mathrm{CN}_{1} \mathrm{HCO}^{+}, \mathrm{H}^{13} \mathrm{CO}+, \mathrm{HNC}^{+}$ and $\mathrm{HN}^{13} \mathrm{C}$, toward M8 $\mathrm{E}$. The square outlined by the dashed red lines in the map of ${ }^{12} \mathrm{CO}$ denotes the area of the smaller maps of $\mathrm{HCN}, \mathrm{H}^{13} \mathrm{CN}$, $\mathrm{HCO}^{+}, \mathrm{H}^{13} \mathrm{CO}^{+}, \mathrm{HNC}$, and $\mathrm{HN}^{13} \mathrm{C}$. Position offsets are relative to the position of M8E-IR (marked with an asterisk) and given in Sect. 2. The contour levels are from 10 to $100 \%$ of the corresponding peak emission (given in Table 1) for ${ }^{12} \mathrm{CO},{ }^{13} \mathrm{CO}, \mathrm{N}_{2} \mathrm{H}^{+}, \mathrm{HCN}, \mathrm{H}^{13} \mathrm{CN}, \mathrm{HCO}^{+}$and $\mathrm{HNC}$. For $\mathrm{H}^{13} \mathrm{CO}^{+}$, and $\mathrm{HN}^{13} \mathrm{C}$ maps, the contour levels are from $3 \mathrm{rms}$ to peak emission in steps of $2 \mathrm{rms}$. All maps are plotted using the original FWHM beam sizes shown in the lower left of each map and listed in Table 1.

that the ATLASGAL contours follow the $\mathrm{N}_{2} \mathrm{H}^{+}$emission distribution, including the secondary emission peak at $\mathrm{N}_{2} \mathrm{H}^{+}$at $(\Delta \alpha, \Delta \delta)=\left(-55^{\prime \prime},-55^{\prime \prime}\right)$, and therefore probe the high-density gas. We ascribe the slight offset $\left(\approx 10^{\prime \prime}\right)$ to the combined position uncertainties of the APEX and our IRAM 30 m telescope observations. Last, to explore the PDR environment, we used the
$8 \mu \mathrm{m}$ data from the archive of the Galactic Legacy Infrared MidPlane Survey Extraordinaire (GLIMPSE, Benjamin et al. 2003; Churchwell et al. 2009) conducted with the Spitzer Space Telescope. The $8 \mu \mathrm{m}$ band of the Infrared Array Camera (IRAC) used by Spitzer traces emission from the polycyclic aromatic hydrocarbons (PAHs), large hydrocarbon molecules (or small 


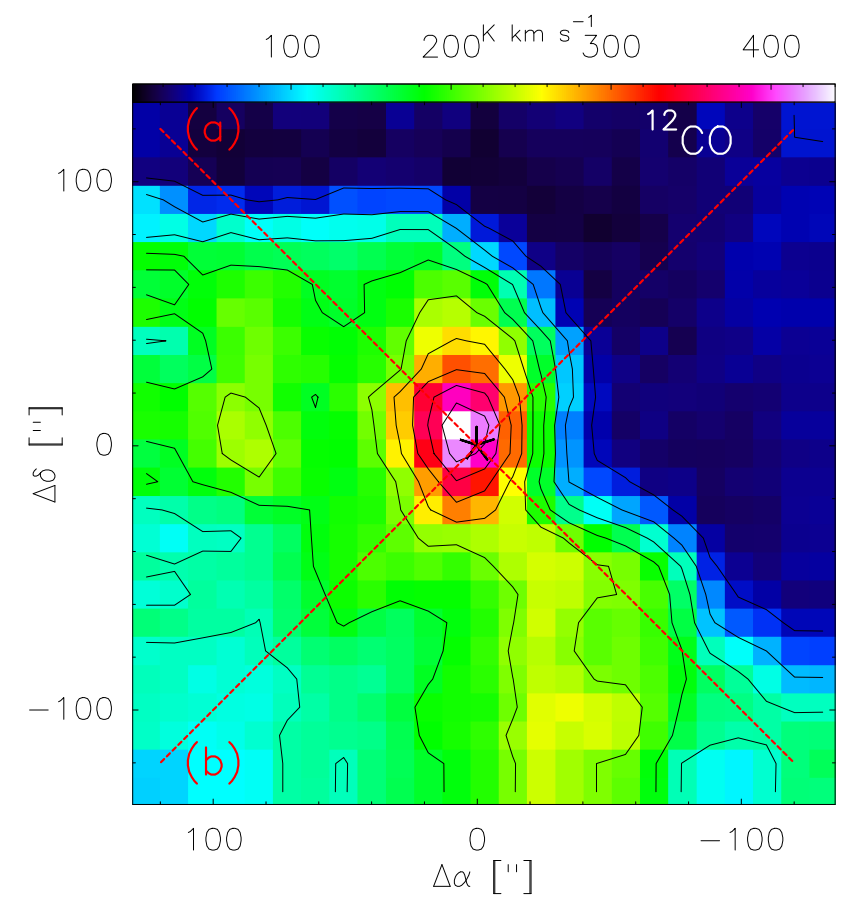

Fig. 4. Velocity-integrated intensity map of ${ }^{12} \mathrm{CO}$ marked with two dashed red cuts: (a) going from $\left(120^{\prime \prime}, 120^{\prime \prime}\right)$ to $\left(-120^{\prime \prime},-120^{\prime \prime}\right)$, and (b) going from $\left(120^{\prime \prime},-120^{\prime \prime}\right)$ to $\left(-120^{\prime \prime}, 120^{\prime \prime}\right)$. Intensity profiles of various species are plotted in these directions in Fig. 5.

dust grains) that become excited by strong UV radiation. The fluorescent IR emission from these PAHs, the result of FUV pumping, arises from the PDR surface of dense molecular clouds and hence traces recent massive star formation activity (Tielens 2008). Figure $6 \mathrm{~d}$ shows the $8 \mu \mathrm{m}$ PAH continuum emission in color scale overlaid with contours of the ${ }^{12} \mathrm{CO}$ gas emission distribution. The high-resolution $8 \mu \mathrm{m}$ emission allows us to probe the bright rimmed structure enclosing the molecular cloud of M8 E. The central H II region of M8 is probably expanding, and this bright rimmed structure corresponds to its ionization front (IF) (discussed further in Sect. 5.2).

\section{Analysis}

We used several complementary methods to determine the temperature and density of the gas that emits the various species observed toward M8 E with the IRAM $30 \mathrm{~m}$ telescope. We start by calculating the excitation temperatures and column densities of ${ }^{12} \mathrm{CO}$ and ${ }^{13} \mathrm{CO}$ using their $J=1 \rightarrow 0$ transitions in Sect. 4.1. In Sect. 4.2 we used multiline data of the dense cloud thermometer $\mathrm{CH}_{3} \mathrm{CCH}$ to determine the temperature of the dense gas, which can then be used to determine the column densities of $\mathrm{N}_{2} \mathrm{H}^{+}, \mathrm{HCN}, \mathrm{H}^{13} \mathrm{CN}, \mathrm{HCO}^{+}, \mathrm{H}^{13} \mathrm{CO}^{+}, \mathrm{HNC}$, and $\mathrm{HN}^{13} \mathrm{C}$ in Sect. 4.3. To complete our investigation of the physical conditions, we undertook nonlocal thermodynamic equilibrium (non-LTE) radiative transfer modeling to constrain the volume densities of the warm and cooler gas of M8 E in Sect. 4.4.

\subsection{Excitation temperature and column density distributions of ${ }^{12} \mathrm{CO}$ and ${ }^{13} \mathrm{CO}$}

When we considered the radiative transfer of the line emission, we assumed that scattering is ignored and that the medium through which the radiation travels is uniform and described by a source function given by the Planck function, with the level populations determined by an excitation temperature, $T_{\mathrm{ex}}$. Assuming the Rayleigh-Jeans approximation and taking the beam filling factor to be unity, we can express the observed main-beam brightness temperature, $T_{\mathrm{mb}}$, in terms of $T_{\mathrm{ex}}$ (Eq. (1) of Peng et al. 2012). A detailed description of radiative transfer relevant here can be found in Mangum \& Shirley (2015). Assuming equal $T_{\text {ex }}$ for ${ }^{12} \mathrm{CO}$ and ${ }^{13} \mathrm{CO}$ and that the ${ }^{12} \mathrm{CO}$ emission is optically thick, the $T_{\mathrm{ex}}$ for the $J=1 \rightarrow 0$ transition of ${ }^{12} \mathrm{CO}$ can be defined as

$T_{\mathrm{mb}}=5.53\left[\frac{1}{\exp \frac{5.53}{T_{\mathrm{ex}}}-1}-\frac{1}{\exp \frac{5.53}{T_{\mathrm{bg}}}-1}\right] \mathrm{K}$.

We took the background temperature, $T_{\mathrm{bg}}$ to be equal to that of the cosmic background radiation, that is, $2.73 \mathrm{~K}$. However, the contribution of the second term within the brackets in Eq. (1) will be $\leq 2 \%$ of $T_{\mathrm{mb}}$ and hence can be neglected. The mainbeam brightness temperature, $T_{\mathrm{mb}}$, is in $\mathrm{K}$ and its values are obtained from the peak $T_{\mathrm{mb}}$ map of ${ }^{12} \mathrm{CO}$ in the velocity range of $\sim 0-20 \mathrm{~km} \mathrm{~s}^{-1}$. The upper panel in Fig. 7 shows the distribution of the resulting $T_{\mathrm{ex}}$ around M8E-IR, peaking in the east and southwest of M8E-IR, with a maximum value of $\sim 85 \pm 1 \mathrm{~K}$.

Under the assumption that the emission from ${ }^{13} \mathrm{CO}$ is optically thin, we used the computed $T_{\mathrm{ex}}$ and the velocity-integrated intensity, $\int T_{\mathrm{mb}}\left({ }^{13} \mathrm{CO}\right) \mathrm{dv}$, to calculate the total column density of ${ }^{13} \mathrm{CO}, N\left({ }^{13} \mathrm{CO}\right)$, by

$$
\begin{aligned}
N\left({ }^{13} \mathrm{CO}\right)= & 4.5 \times 10^{13}\left(T_{\mathrm{ex}}+0.88\right) \exp \left(\frac{5.3}{T_{\mathrm{ex}}}\right) \\
& \times \int T_{\mathrm{mb}}\left({ }^{13} \mathrm{CO}\right) \mathrm{dv} \mathrm{cm}^{-2},
\end{aligned}
$$

where $T_{\mathrm{ex}}$ is in $\mathrm{K}$ and $\int T_{\mathrm{mb}}\left({ }^{13} \mathrm{CO}\right) \mathrm{dv}$ is in $\mathrm{K} \mathrm{km} \mathrm{s}^{-1}$. The resulting $N\left({ }^{13} \mathrm{CO}\right)$ distribution is shown in Fig. 7 (lower panel), peaking at M8E-IR with a maximum value of $\sim 3.0 \times 10^{17} \mathrm{~cm}^{-2}$. We calculated the $\mathrm{H}_{2}$ column density, $N\left(\mathrm{H}_{2}\right)$, with a peak value of $\sim 1.5 \times 10^{23} \mathrm{~cm}^{-2}$, by adopting an isotopic ratio of $\left[\mathrm{CO} /{ }^{13} \mathrm{CO}\right] \sim$ 45, appropriate for the Galactocentric distance of M8 (Milam et al. 2005) and a ${ }^{12} \mathrm{CO}$ abundance ratio of $\left[\mathrm{CO} / \mathrm{H}_{2}\right] \sim 8.5 \times 10^{-5}$ (Tielens 2010).

\section{2. $\mathrm{CH}_{3} \mathrm{CCH}$ dense gas thermometry}

Methyl acetylene, $\mathrm{CH}_{3} \mathrm{CCH}$, was first detected by Snyder \& Buhl (1973) in Sgr B2. It is a symmetric top molecule with quantum numbers, $J$ and $K$, which define the total angular momentum and its projection along the symmetry axis, respectively. For each energy level with total angular momentum $J$ there are is a "ladder" of $J$ different $K$ states with quantum numbers $0<K<$ $J-1$. The states in each $K$ ladder span a wide range in energies above the ground, while the frequencies in the resulting lines lie very close to each other. The electric dipole moment of $\mathrm{CH}_{3} \mathrm{CCH}$ is parallel to the symmetry axis, so that the only possible $J \rightarrow$ $J-1$ transitions have $\Delta K=0$. For a given $J$ level, the different $K$ levels are populated only through collisions, and the total population in each $K$ ladder therefore is a function of only the kinetic temperature of the dense gas emitting the $\mathrm{CH}_{3} \mathrm{CCH}$ lines. The temperature can be determined from the (measured) relative level populations. This makes $\mathrm{CH}_{3} \mathrm{CCH}$ an excellent molecular cloud thermometer (e.g., Bergin et al. 1994 and Giannetti et al. 2012, 2017). 

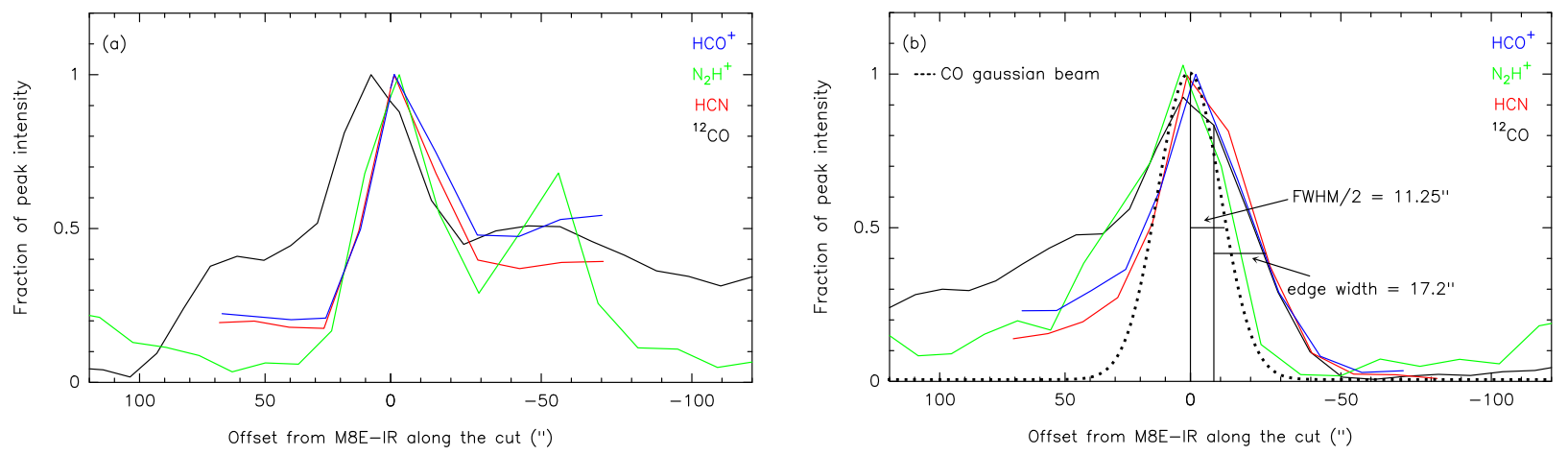

Fig. 5. Velocity-integrated intensities normalized to their value at peak vs. offset (") from M8E-IR along the cuts shown in Fig. 4.
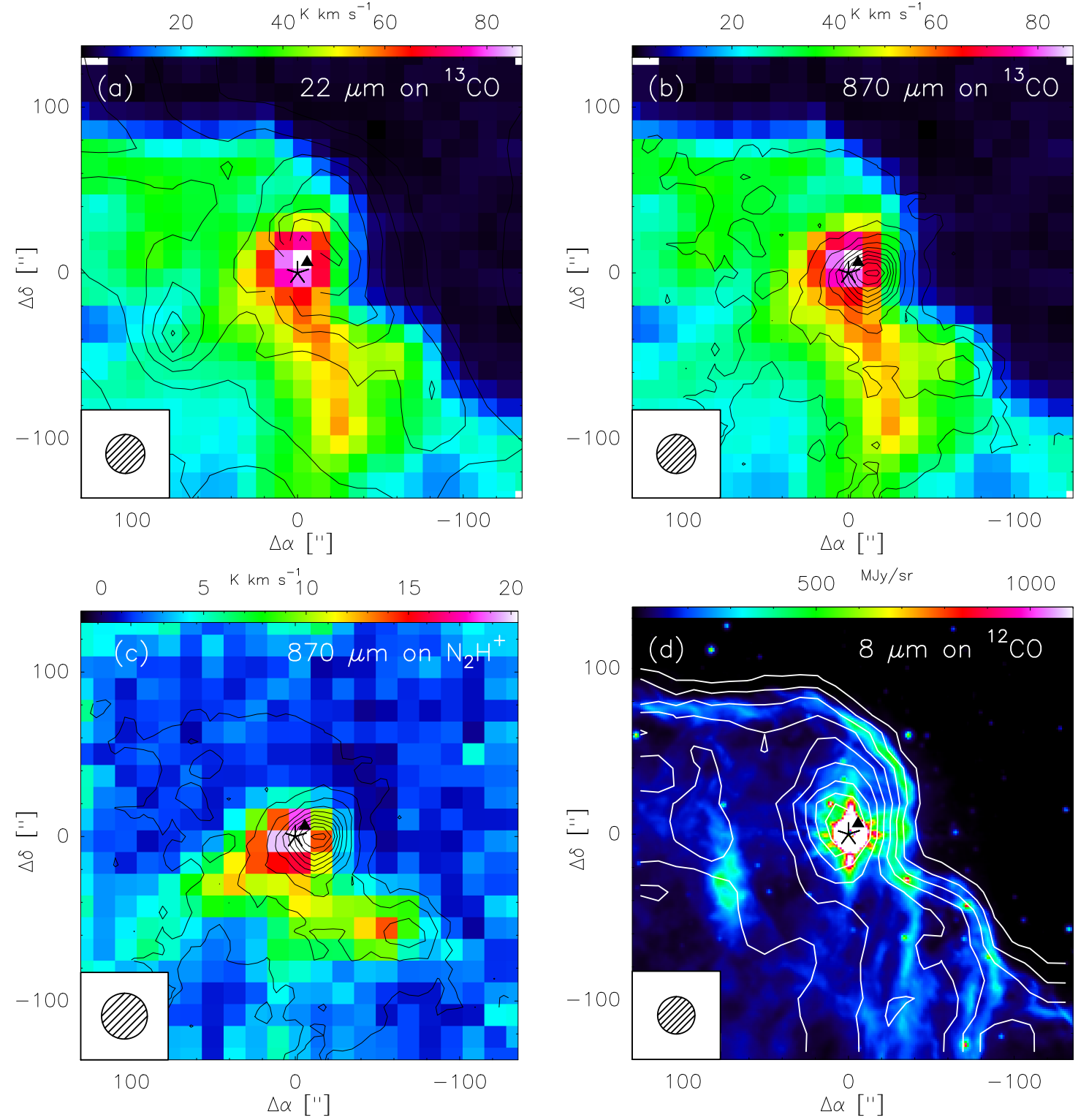

Fig. 6. Top row: color map of the velocity-integrated intensity of $J=1 \rightarrow 0$ transition of ${ }^{13} \mathrm{CO}$ overlaid with contours of panel $a$, WISE $22 \mu \mathrm{m}$ midIR continuum emission and (b) ATLASGAL $870 \mu \mathrm{m}$ continuum emission toward M8 E. Bottom row, panel $c$ : color map of the velocity-integrated intensity of $J=1 \rightarrow 0$ transition of $\mathrm{N}_{2} \mathrm{H}^{+}$overlaid with ATLASGAL $870 \mu \mathrm{m}$ dust continuum emission, and panel $d$ : contours of the velocityintegrated intensity map of ${ }^{12} \mathrm{CO}$ overlaid on the $8 \mu \mathrm{m}$ PAH emission map. Position offsets are relative to the position of M8E-IR (marked with an asterisk) given in Sect. 2, and the ultracompact H II region, M8E-radio, at $(\alpha, \delta)_{\mathrm{J} 2000}=18^{\mathrm{h}} 04^{\mathrm{m}} 52^{\mathrm{s}} 8,-24^{\circ} 26^{\prime} 36^{\prime \prime}$ is represented by a black triangle. The ${ }^{12} \mathrm{CO}$ and ${ }^{13} \mathrm{CO}$ emission maps have their original resolutions of $22.5^{\prime \prime}$ and $23.5^{\prime \prime}$, respectively,the MIPSGAL $24 \mu \mathrm{m}$ continuum emission has a resolution of $6^{\prime \prime}$, the ATLASGAL $870 \mu$ m continuum emission has a resolution of $18.2^{\prime \prime}$, and the GLIMPSE $8 \mu$ m continuum emission has a resolution of $0.6^{\prime \prime}$. The contour levels are 5 to $100 \%$ in steps of $10 \%$ for the MIPSGAL $24 \mu \mathrm{m}(a)$ and the ATLASGAL $870 \mu \mathrm{m}(b, c)$ overlays. For the GLIMPSE $8 \mu \mathrm{m}(d)$ overlay, the contour levels are 10 to $100 \%$ in steps of $10 \%$. 

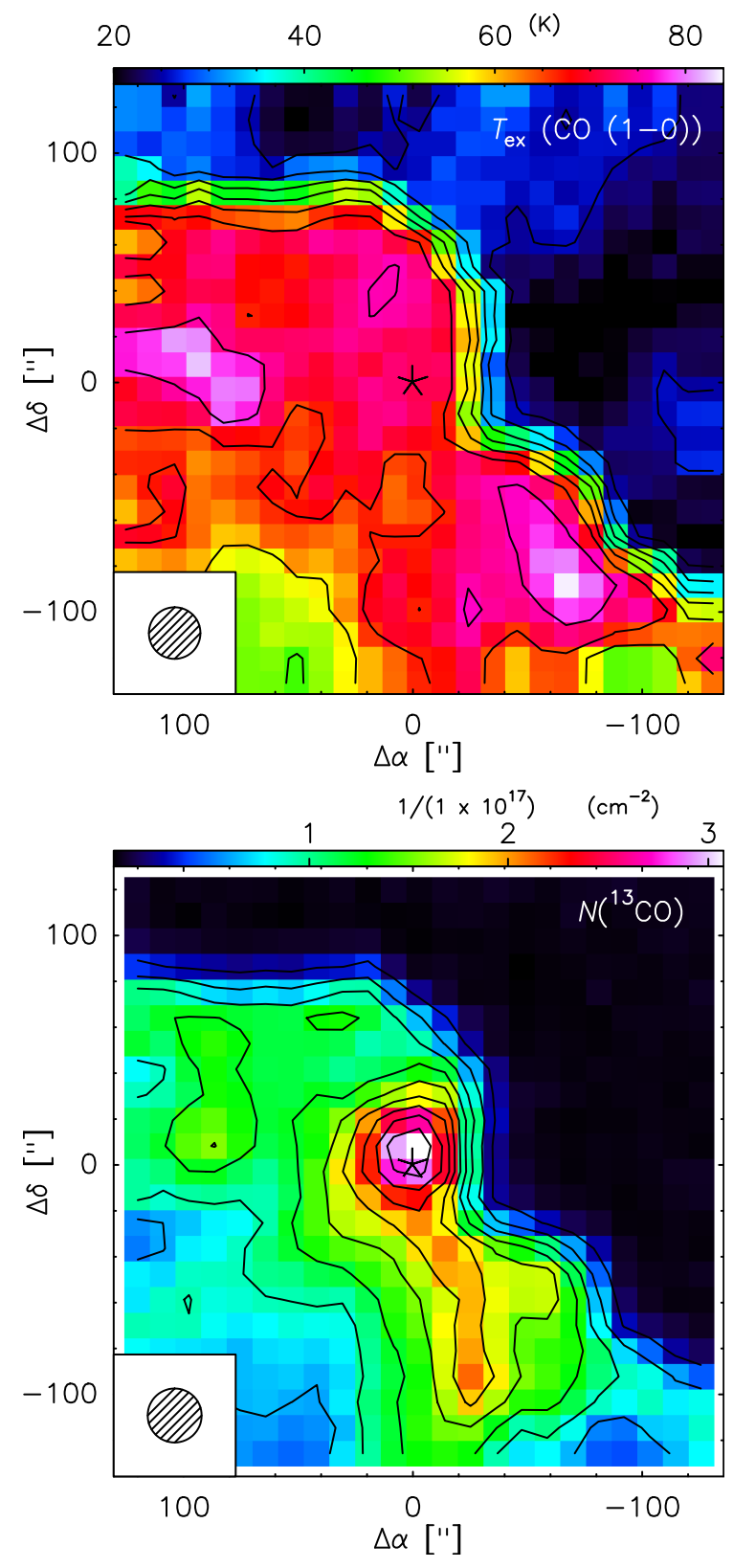

Fig. 7. Upper panel: excitation temperature $T_{\text {ex }}$ determined for the $J=$ $1 \rightarrow 0$ transition of ${ }^{12} \mathrm{CO}$ and ${ }^{13} \mathrm{CO}$. Lower panel: total column density of ${ }^{13} \mathrm{CO}, N\left({ }^{13} \mathrm{CO}\right)$. Position offsets are relative to the position of M8EIR (marked with an asterisk) given in Sect. 2. The contour levels are from 10 to $100 \%$ in steps of $10 \%$ peak emission (Table 1). The values of $T_{\mathrm{mb}}$ and that of $\int T_{\mathrm{mb}}\left({ }^{13} \mathrm{CO}\right) \mathrm{dv}$ for the $J=1 \rightarrow 0$ transition used to calculate $T_{\text {ex }}$ and $N\left({ }^{13} \mathrm{CO}\right)$ were extracted from maps convolved to the same resolution of $24^{\prime \prime}$

To derive the dense gas temperature, we simultaneously fit (as shown in Fig. 8) the $J=5 \rightarrow 4$ and $6 \rightarrow 5$ transitions of $\mathrm{CH}_{3} \mathrm{CCH}$ observed toward M8E-IR. We used MCWeeds (Giannetti et al. 2017), which provides an external interface between Weeds (a class extension for the analysis of multimolecule/ multitransition millimeter and submillimeter spectral surveys, Maret et al. 2011) and PyMC $^{5}$ (a python package for efficiently coding a probabilistic model and draw samples from its posterior distribution using Markov chain Monte Carlo techniques, Patil et al. 2010). Weeds generates synthetic spectra by

\footnotetext{
https://pymc-devs.github.io/pymc/index.html
}

solving the radiative transfer equation assuming LTE and takes the finite angular resolution of the observations into account, but does not optimize algorithms. On the other hand, PyMC implements Bayesian statistical models and fit algorithms. Initial guesses are given for a set of input parameters along with their probability distribution and the range over which they should be varied. By considering multiple transitions of a molecule, McWeeds provides best-fit parameters with their errors (for more details, see Giannetti et al. 2017 and Thiel et al. 2019).

To fit the spectrum, an input file is provided containing all the initial guesses of the parameters: column density $\left(\mathrm{cm}^{-2}\right)$, temperature $(\mathrm{K})$, size of the emitting region (arcsec), radial velocity $\left(\mathrm{km} \mathrm{s}^{-1}\right)$ and line width $\left(\mathrm{km} \mathrm{s}^{-1}\right)$, rms noise $(\mathrm{K})$, and calibration uncertainty for the spectral range. The input file also contains the name of the species, the frequency range in which the interested transition lies, the corresponding data file, and the priors required to fit the observed spectrum. Using the ATLASGAL $870 \mu \mathrm{m}$ image, we estimated the source size $\sim 40^{\prime \prime}$, which is larger than the beam size $\left(\sim 30^{\prime \prime}\right)$ with which $\mathrm{CH}_{3} \mathrm{CCH}$ lines were observed. Therefore, we fixed the size of the emitting region to $30^{\prime \prime}$. Similar to Giannetti et al. (2017), we used loosely informative priors for the parameters of the fit: a normal Gaussian function for column densities and velocities, and a truncated normal Gaussian function is used for temperatures and line widths. The probability distributions of these functions depend on the values of their mean, $\mu$, and standard deviation, $\sigma$ (a detailed description of priors can be found in Jaynes 2003).

The given input (initial guesses along with their upper and lower bounds) and the obtained output values of the fit parameters used in MCWeeds are listed in Table 3. The resulting dense gas temperature is $32.9 \pm 1.4 \mathrm{~K}$, which is in excellent agreement with the value for the kinetic temperature of the molecular gas toward M8 E $(31 \mathrm{~K})$ determined from measurements of $\mathrm{NH}_{3}$ emission with a comparable beam size $\left(40^{\prime \prime} F W H M\right)$ (Molinari et al. 1996). A total $\mathrm{CH}_{3} \mathrm{CCH}$ column density, $N\left(\mathrm{CH}_{3} \mathrm{CCH}\right)$, of $(5.3 \pm 0.1) \times 10^{14} \mathrm{~cm}^{-2}$ was obtained.

\subsection{Column density estimates of $\mathrm{N}_{2} \mathrm{H}^{+}, \mathrm{HCN}, \mathrm{HCO}^{+}, \mathrm{HNC}$, $\mathrm{H}^{13} \mathrm{CN}, \mathrm{H}^{13} \mathrm{CO}^{+}$, and $\mathrm{HN}^{13} \mathrm{C}$}

In Sects. 4.1 and 4.2, we estimated the temperatures of the warm, low-density and the cooler higher density gas components based on ${ }^{12} \mathrm{CO}$ and $\mathrm{CH}_{3} \mathrm{CCH}$ emission, respectively. We assumed that the temperature of the cool gas $(\sim 32 \mathrm{~K})$ estimated from $\mathrm{CH}_{3} \mathrm{CCH}$ is similar to the kinetic temperature of the gas that emits $\mathrm{N}_{2} \mathrm{H}^{+}, \mathrm{HCN}, \mathrm{HCO}^{+}, \mathrm{HNC}, \mathrm{H}^{13} \mathrm{CN}, \mathrm{H}^{13} \mathrm{CO}^{+}$, and $\mathrm{HN}^{13} \mathrm{C}$. Thus we calculated the total column densities of these molecules by fitting the synthetic spectra generated by MCWeeds to the observed spectra of their $J=1 \rightarrow 0$ transition toward M8E-IR, keeping temperature and size fixed. The input and output parameters are listed in Table 3. The calculated column densities of $\mathrm{HCN}, \mathrm{HCO}^{+}$and $\mathrm{HNC}$ should be considered as upper limits to the real values as we assumed the cool gas temperature for their evaluation. We compared our results with a similar survey done toward W 51, where Watanabe et al. (2017) assumed lower excitation temperatures $(\leq 20 \mathrm{~K})$, and we find that the total column densities determined for the observed species in M8 E are relatively higher.

\subsection{Non-LTE analysis}

In Sects. 4.1-4.3 we used several techniques under the assumption of LTE to determine the column densities of the observed 
Table 3. Priors, given input, and resulting output from the best-fit models.

\begin{tabular}{|c|c|c|c|c|}
\hline & Temperature (K) & Column density $\left(\log \left(\mathrm{cm}^{-2}\right)\right)$ & Line width $\left(\mathrm{km} \mathrm{s}^{-1}\right)$ & Velocity $\left(\mathrm{km} \mathrm{s}^{-1}\right)$ \\
\hline \multicolumn{5}{|c|}{$\mathrm{CH}_{3} \mathrm{CCH} J=5 \rightarrow 4$ and $6 \rightarrow 5$} \\
\hline $\begin{array}{l}\text { Prior } \\
\text { Input }\end{array}$ & $\begin{array}{c}\text { Truncated normal } \\
\qquad \begin{array}{c}\mu=50 \\
\sigma=30\end{array} \\
\text { low }=10, \text { high }=80\end{array}$ & $\begin{array}{c}\text { Normal } \\
\mu=14 \\
\sigma=2\end{array}$ & $\begin{array}{l}\text { Truncated normal } \\
\qquad \begin{array}{l}\mu=5 \\
\sigma=3\end{array} \\
\text { low }=1, \text { high }=35\end{array}$ & $\begin{array}{c}\text { Normal } \\
\mu=0 \\
\sigma=2\end{array}$ \\
\hline Output & $32.9(1.4)$ & $14.72(0.047)$ & $2.03(0.05)$ & $-0.24(0.019)$ \\
\hline \multicolumn{5}{|c|}{$\mathrm{N}_{2} \mathrm{H}^{+} J=1 \rightarrow 0$} \\
\hline $\begin{array}{l}\text { Prior } \\
\text { Input }\end{array}$ & $\begin{array}{c}\text { Fixed } \\
\text { value }=33\end{array}$ & $\begin{array}{c}\text { Normal } \\
\mu=14 \\
\sigma=2\end{array}$ & $\begin{array}{l}\text { Truncated normal } \\
\qquad \begin{array}{l}\mu=5 \\
\sigma=3\end{array} \\
\text { low }=1, \text { high }=35\end{array}$ & $\begin{array}{c}\text { Normal } \\
\mu=0 \\
\sigma=2\end{array}$ \\
\hline Output & 33 & $14.11(0.042)$ & $2.24(0.03)$ & $-0.28(0.01)$ \\
\hline \multicolumn{5}{|c|}{$\mathrm{HCN} J=1 \rightarrow 0$} \\
\hline $\begin{array}{l}\text { Prior } \\
\text { Input }\end{array}$ & $\begin{array}{c}\text { Fixed } \\
\text { value }=33\end{array}$ & $\begin{array}{c}\text { Normal } \\
\mu=14 \\
\sigma=2\end{array}$ & $\begin{array}{l}\text { Truncated normal } \\
\qquad \begin{array}{l}\mu=5 \\
\sigma=3\end{array} \\
\text { low }=1, \text { high }=35\end{array}$ & $\begin{array}{l}\text { Normal } \\
\mu=0 \\
\sigma=2\end{array}$ \\
\hline Output & 33 & $15.24(0.042)$ & $3.07(0.08)$ & $-0.37(0.03)$ \\
\hline \multicolumn{5}{|c|}{$\mathrm{H}^{13} \mathrm{CN} J=1 \rightarrow 0$} \\
\hline $\begin{array}{l}\text { Prior } \\
\text { Input }\end{array}$ & $\begin{array}{c}\text { Fixed } \\
\text { value }=33\end{array}$ & $\begin{array}{c}\text { Normal } \\
\mu=14 \\
\sigma=2\end{array}$ & $\begin{array}{l}\text { Truncated normal } \\
\qquad \begin{array}{l}\mu=5 \\
\sigma=3\end{array} \\
\text { low }=1, \text { high }=35\end{array}$ & $\begin{array}{l}\text { Normal } \\
\mu=0 \\
\sigma=2\end{array}$ \\
\hline Output & 33 & $13.84(0.037)$ & $2.25(0.02)$ & $-0.22(0.008)$ \\
\hline \multicolumn{5}{|c|}{$\mathrm{HCO}^{+} J=1 \rightarrow 0$} \\
\hline $\begin{array}{l}\text { Prior } \\
\text { Input }\end{array}$ & $\begin{array}{c}\text { Fixed } \\
\text { value }=33\end{array}$ & $\begin{array}{c}\text { Normal } \\
\mu=14 \\
\sigma=2\end{array}$ & $\begin{array}{l}\text { Truncated normal } \\
\qquad \begin{array}{l}\mu=5 \\
\sigma=3\end{array} \\
\text { low }=1, \text { high }=35\end{array}$ & $\begin{array}{c}\text { Normal } \\
\begin{array}{l}\mu=0 \\
\sigma=2\end{array}\end{array}$ \\
\hline Output & 33 & $14.84(0.027)$ & $2.45(0.038)$ & $-0.13(0.009)$ \\
\hline \multicolumn{5}{|c|}{$\mathrm{H}^{13} \mathrm{CO}^{+} J=1 \rightarrow 0$} \\
\hline $\begin{array}{l}\text { Prior } \\
\text { Input }\end{array}$ & $\begin{array}{c}\text { Fixed } \\
\text { value }=33\end{array}$ & $\begin{array}{c}\text { Normal } \\
\mu=14 \\
\sigma=2\end{array}$ & $\begin{array}{l}\text { Truncated normal } \\
\qquad \begin{array}{c}\sigma=5 \\
\sigma=3\end{array} \\
\text { low }=1, \text { high }=35\end{array}$ & $\begin{array}{c}\text { Normal } \\
\mu=0 \\
\sigma=2\end{array}$ \\
\hline Output & 33 & $12.97(0.045)$ & $2.1(0.03)$ & $-0.23(0.01)$ \\
\hline \multicolumn{5}{|c|}{$\mathrm{HNC} J=1 \rightarrow 0$} \\
\hline $\begin{array}{l}\text { Prior } \\
\text { Input }\end{array}$ & $\begin{array}{c}\text { Fixed } \\
\text { value }=33\end{array}$ & $\begin{array}{c}\text { Normal } \\
\mu=14 \\
\sigma=2\end{array}$ & $\begin{array}{l}\text { Truncated normal } \\
\qquad \begin{array}{c}\mu=5 \\
\sigma=3\end{array} \\
\text { low }=1, \text { high }=35\end{array}$ & $\begin{array}{c}\text { Normal } \\
\mu=0 \\
\sigma=2\end{array}$ \\
\hline Output & 33 & $14.35(0.05)$ & $2.59(0.032)$ & $-0.12(0.007)$ \\
\hline \multicolumn{5}{|c|}{$\mathrm{HN}^{13} \mathrm{C} J=1 \rightarrow 0$} \\
\hline $\begin{array}{l}\text { Prior } \\
\text { Input }\end{array}$ & $\begin{array}{c}\text { Fixed } \\
\text { value }=33\end{array}$ & $\begin{array}{c}\text { Normal } \\
\mu=14 \\
\sigma=2\end{array}$ & $\begin{array}{l}\text { Truncated normal } \\
\qquad \begin{array}{c}\mu=5 \\
\sigma=3\end{array} \\
\text { low }=1, \text { high }=35\end{array}$ & $\begin{array}{c}\text { Normal } \\
\mu=0 \\
\sigma=2\end{array}$ \\
\hline Output & 33 & $13.24(0.04)$ & $2.1(0.043)$ & $-0.06(0.01)$ \\
\hline
\end{tabular}



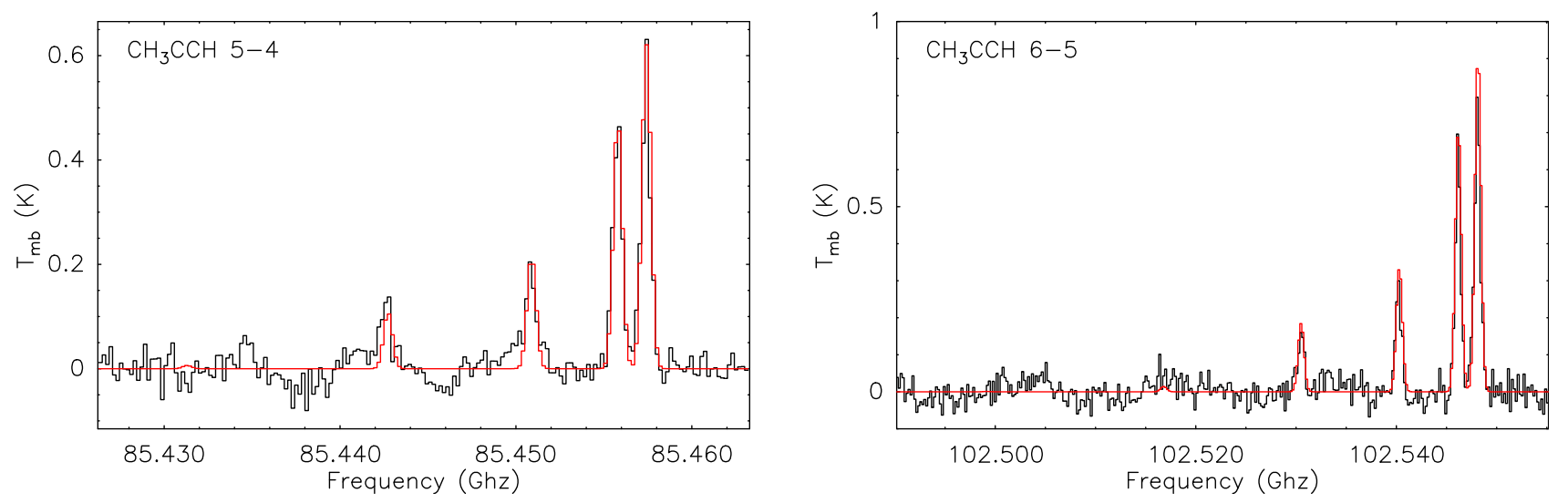

Fig. 8. Spectra of the $J=5 \rightarrow 4$ and $6 \rightarrow 5$ transitions of $\mathrm{CH}_{3} \mathrm{CCH}$ observed toward M8E-IR. In red we plot the best-fit synthetic spectra generated by MCWeeds for the input parameters listed in Table 3.

species. To determine how much these values deviate under nonLTE conditions, we used RADEX (van der Tak et al. 2007), which is a non-LTE radiative transfer program.

RADEX is a computer code for performing statistical equilibrium calculations that treats the radiative transfer using the escape probability approximation in an isothermal and homogeneous medium, taking optical depth effects into account. We selected a uniform spherical geometry, and the collisional rate coefficients required for the modeling were taken from the Leiden Molecular and Atomic Database ${ }^{6}$ (LAMDA; Schöier et al. 2005). With the exception of $\mathrm{H}^{13} \mathrm{CN}, \mathrm{CH}_{3} \mathrm{CCH}$, and $\mathrm{HN}^{13} \mathrm{C}$, LAMDA provides collisional rate coefficients for all species analyzed in this paper. For ${ }^{12} \mathrm{CO}$ and ${ }^{13} \mathrm{CO}$, the collisional rate coefficients with $\mathrm{H}_{2}$ were adopted from Yang et al. (2010). The $\mathrm{N}_{2} \mathrm{H}^{+}-\mathrm{H}_{2}$ collisional rate coefficients were calculated (see Schöier et al. 2005) from the $\mathrm{N}_{2} \mathrm{H}^{+}-\mathrm{He}$ collisional rate coefficients given by Daniel et al. (2005) for hfs components. The $\mathrm{HCN}-\mathrm{H}_{2}$ collisional rate coefficients have been calculated by Hernández Vera et al. (2017) and the coefficients for the hfs components were computed following Braine et al. (in prep.). The $\mathrm{H}^{13} \mathrm{CO}^{+}-\mathrm{H}_{2}$ collisional rate coefficients were extrapolated from the $\mathrm{HCO}^{+}-\mathrm{H}_{2}$ collisional rate coefficients, which are provided by Flower (1999). The $\mathrm{HNC}-\mathrm{H}_{2}$ collisional rate coefficients were calculated by multiplying the $\mathrm{HNC}-\mathrm{He}$ collision rates given by Dumouchel et al. (2010) by a factor of 1.37.

For the di- and triatomics we only have data for a single transition each, therefore we cannot constrain the volume densities, and we were guided by the concepts of critical, $n_{\mathrm{cr}}$, and effective, $n_{\mathrm{eff}}$, densities as estimates for the densities required to excite the observed lines. Shirley (2015) thoroughly compared the relevance of $n_{\mathrm{cr}}$ and $n_{\mathrm{eff}}$. The common usage of critical density $n_{\mathrm{cr}}$ applies for the optically thin limit and ignores radiative trapping (Shirley 2015). In particular for ground state lines, the line opacities can be very high. They should therefore only be considered as an upper limit for the density needed to excite the rotational levels of the (Pety et al. 2017). For optically thick lines, the effective density becomes relevant. Using the ${ }^{12} \mathrm{C} /{ }^{13} \mathrm{C} \sim 45$ (Milam et al. 2005), we calculated the optical depths $\tau_{{ }^{12}} \mathrm{CO} \sim 19$, $\tau_{\mathrm{HCN}} \sim 7, \tau_{\mathrm{HCO}^{+}} \sim 6$ and $\tau_{\mathrm{HNC}}=3.9$. For $\mathrm{N}_{2} \mathrm{H}^{+}$, we fit its different hfs components using the Class software and obtained $\tau_{\mathrm{N}_{2} \mathrm{H}^{+}} \sim$ 0.6 . For optically thick lines, $n_{\mathrm{eff}}=n_{\mathrm{cr}} \times(1-\exp (-\tau)) / \tau$ (Shirley 2015).

\footnotetext{
6 https://home.strw.leidenuniv.nl/ moldata/
}

Table 4. Comparison of column densities determined from RADEX and LTE analyses.

\begin{tabular}{ccccc}
\hline \hline \multirow{2}{*}{ Species } & \multicolumn{3}{c}{ non-LTE $(\mathrm{RADEX})$} & $\begin{array}{c}\text { LTE } \\
\end{array}$ \\
\cline { 2 - 4 } & $T_{\mathrm{k}}(\mathrm{K})$ & $n\left(\mathrm{H}_{2}\right)\left(\mathrm{cm}^{-3}\right)$ & $N(\mathrm{X})\left(\mathrm{cm}^{-2}\right)$ & $N(\mathrm{X})\left(\mathrm{cm}^{-2}\right)$ \\
\hline${ }^{12} \mathrm{CO}$ & 80 & $10^{4}$ & $7.8 \times 10^{18}$ & $1.3 \times 10^{19}$ \\
${ }^{13} \mathrm{CO}$ & 80 & $10^{4}$ & $3.6 \times 10^{17}$ & $2 \times 10^{17}$ \\
$\mathrm{~N}_{2} \mathrm{H}^{+}$ & 30 & $3.710^{5}$ & $1 \times 10^{14}$ & $5.2 \times 10^{14}$ \\
$\mathrm{HCN}^{3}$ & 30 & $1.5 \times 10^{5}$ & $1 \times 10^{15}$ & $1.7 \times 10^{15}$ \\
$\mathrm{HCO}^{+}$ & 30 & $3.2 \times 10^{4}$ & $5 \times 10^{14}$ & $7 \times 10^{14}$ \\
$\mathrm{H}^{13} \mathrm{CO}^{+}$ & 30 & $1.8 \times 10^{5}$ & $7 \times 10^{12}$ & $9.3 \times 10^{12}$ \\
$\mathrm{HNC}^{12}$ & 30 & $8 \times 10^{4}$ & $4.2 \times 10^{14}$ & $2.2 \times 10^{14}$ \\
\hline
\end{tabular}

Assuming a background temperature of $2.73 \mathrm{~K}$ and taking the line width values of the above mentioned species from the observed spectra (toward M8E-IR), we ran the models for fixed kinetic temperatures and volume densities, while varying the column densities to reach the intensities that best fit our observed values. Kinetic temperatures of 80 and $30 \mathrm{~K}$ were used as derived above from ${ }^{12} \mathrm{CO}$ and $\mathrm{CH}_{3} \mathrm{CCH}$, respectively. We set the volume densities (given in Table 4) to the same values as $n_{\text {cr }}$ for optically thin species $\left({ }^{13} \mathrm{CO}, \mathrm{H}^{13} \mathrm{CO}^{+}\right.$, and $\left.\mathrm{N}_{2} \mathrm{H}^{+}\right)$and $n_{\text {eff }}$ for optically thick species ( ${ }^{12} \mathrm{CO}, \mathrm{HCN}, \mathrm{HCO}^{+}$, and $\mathrm{HNC}$ ).

As mentioned in Sect. 4.2, the LTE analysis was made for a fixed beam size of $30^{\prime \prime}$ and the source size is $\sim 40^{\prime \prime}$. To compare the RADEX modeling results with the LTE analysis, we therefore divided the column density values predicted by RADEX by a resulting beam-filling factor of 0.64 , and these results are reported in Table 4 . For ${ }^{12} \mathrm{CO}$, with a kinetic temperature of $80 \mathrm{~K}$ and a $n_{\text {eff }} \sim 10^{2} \mathrm{~cm}^{-3}$, the models do not reach the peak intensity of $\sim 72 \mathrm{~K}$. Volume densities $>10^{4} \mathrm{~cm}^{-3}$ are required in order to reach the observed peak intensity. The corresponding column density is given in Table 4. For consistency, we used a temperature of $80 \mathrm{~K}$ and volume density of $10^{4} \mathrm{~cm}^{-3}$ for ${ }^{13} \mathrm{CO}$ as well. At lower densities $\left(n_{\mathrm{cr}}=2 \times 10^{3} \mathrm{~cm}^{-3}\right)$, the column density values are much higher than estimated by the LTE analysis. The column density has an inverse dependence on $n_{\text {eff }}$ (Shirley 2015). Assuming higher volume densities than $n_{\text {cr }}$ and $n_{\text {eff }}$ can also be justified, given that we detect lines from higher density probes such as $\mathrm{N}_{2} \mathrm{H}^{+}$toward M8 E. For the remaining species, we obtained column densities similar to those calculated from the LTE analysis. 


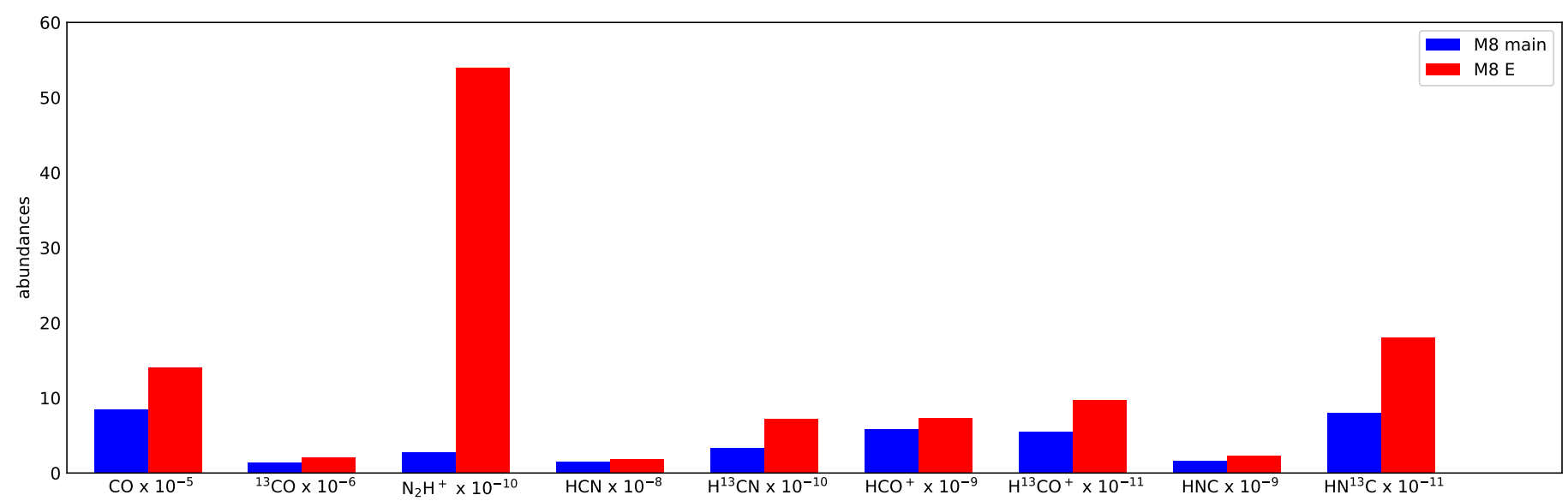

Fig. 9. Chemical abundances of ${ }^{12} \mathrm{CO},{ }^{13} \mathrm{CO}, \mathrm{HCN}, \mathrm{H}^{13} \mathrm{CN}, \mathrm{HCO}^{+}, \mathrm{H}^{13} \mathrm{CO}^{+}, \mathrm{HNC}$ and $\mathrm{HN}^{13} \mathrm{C}$ observed toward $\mathrm{M} 8 \mathrm{E}$ (red bars) and $\mathrm{M} 8-\mathrm{Main}$ (blue bars).

\section{Discussion}

\subsection{Comparison with M8-Main}

Because of the proximity of M8 E to M8-Main and because the two compete at IR and submm wavelengths (Tothill et al. 2008), it is only natural to compare the two regions based on their chemical abundances and physical conditions. A comparison of the abundances, $N(\mathrm{X}) / N\left(\mathrm{H}_{2}\right)$, of various species observed toward M8-Main and M8 E is presented in Fig. 9. For M8 E, the abundances were calculated from the column densities of various species, $N(\mathrm{X})$, obtained from the LTE analysis reported in Sects. 4.1-4.3, while the $\mathrm{H}_{2}$ column density was calculated using the intensity of $870 \mu \mathrm{m}$ continuum emission measured in the ATLASGAL survey, which is $5588 \mathrm{mJy} \mathrm{beam}^{-1}$ toward M8E-IR, and by assuming the dust temperature, $T_{\mathrm{d}}$, of $28.6 \mathrm{~K}$ determined by Tothill et al. (2002). We assumed a gas-to-dust mass ratio of 100 and an absorption coefficient of $1.85 \mathrm{~g}^{-1} \mathrm{~cm}^{2}$ (Schuller et al. 2009). Using Eq. (A.27) from Kauffmann et al. (2008), we calculated a peak molecular hydrogen column density, $N\left(\mathrm{H}_{2}\right)$, of $1.46 \times 10^{22} \mathrm{~cm}^{-2}$, which agrees reasonably well with the values discussed in Sect. 4.1: it agrees to within a factor $\sim 1.5$. For M8-Main we computed the column densities toward Her 36, which is the brightest LOS (similar to M8E-IR in M8 E). We adopted the ${ }^{12} \mathrm{CO}$ and ${ }^{13} \mathrm{CO}$ column densities as determined from the LTE analysis in Tiwari et al. (2018, Sect. 4.1), and for the other molecules analyzed in the work, we used the same techniques as mentioned in Sects. 4.2 and 4.3. A full description of the $3 \mathrm{~mm}$ observations toward M8-Main is given in Tiwari et al. (2019, Sect. 2.2).

Figure 9 shows in general, the abundance of each species is found to be higher toward M8 E than in M8-Main. Although for most of the molecules the difference is small, for $\mathrm{N}_{2} \mathrm{H}^{+}$and $\mathrm{HN}^{13} \mathrm{C}$, the abundances found toward M8 E are about 25 times and 2 times larger, respectively, than in M8-Main. Because the $J=1 \rightarrow 0$ transition of $\mathrm{N}_{2} \mathrm{H}^{+}$has been argued to be a better tracer of very dense gas, which is not relatively bright in M8Main, compared to the other traditional molecules such as $\mathrm{HCN}$ and $\mathrm{HCO}^{+}$(e.g., Kauffmann et al. 2017, Pety et al. 2017 and Brinkmann et al. 2020), we infer that in M8 E, star formation occurs in a densely embedded molecular cloud core that has no counterpart in M8-Main, which in general represents a warmer and more diffuse PDR-dominated environment. This is consistent with obvious picture that the molecular cloud of M8 E, powered by M8E-IR, which will soon emerge as BO-type star, is at an earlier stage of star formation than M8-Main, in which more massive young stellar objects, represented by the Her 36 system and other O-type stars, already excite a prominent H II region.

\subsection{Spatial structure relative to the ionization front of $M 8$}

The large-scale structure of the Lagoon nebula has been discussed based on optical, radio, and IR data by Lada et al. (1976), Woodward et al. (1986), and Tothill et al. (2008), and the zoomed-in view of the M8-Main region by Tiwari et al. (2018). When we combine our previous knowledge of the Lagoon nebula and the information we attained from analyzing the observed spatial distribution of the molecular line emission toward M8 E in Sects. 3.1 and 3.2, the picture emerges that M8 E is situated at the edge of the nebula, formed due to the material being pushed away from the central H II region as the IF (shown in Fig. 10) is moving to the southeast. Figures 3 and $6 \mathrm{~d}$ allow a comparison of the spatial variation of our observed species with respect to the IF. Of all the species discussed in this work, the ${ }^{12} \mathrm{CO}$ and ${ }^{13} \mathrm{CO}$ trace the IF best, and $N_{2} \mathrm{H}^{+}$traces it least well. Furthermore, $\mathrm{HCN}, \mathrm{HCO}^{+}$, and $\mathrm{HNC}$ emission trace the immediate boundary of the IF both in the north and south, but this trend is not followed by their ${ }^{13} \mathrm{C}$ isotopes. Although $\mathrm{H}^{13} \mathrm{CN}, \mathrm{H}^{13} \mathrm{CO}^{+}$, and $\mathrm{HN}^{13} \mathrm{C}$ emission trace the southern boundary of the IF, they show no emission toward the northern boundary of the IF. We interpret this to mean that the gas south of M8E-IR is denser than the gas north of it.

\subsection{Compression of $M 8 E$ and possible triggered star formation?}

The expansion of the IF is expected to cause compressing and heating in the part of the molecular cloud that faces it, which causes the emission of molecular lines as reported in this work. Based on our findings about the IF (bright rimmed structure shown in Fig. 6d) and about M8 E environment being denser compared to M8-Main, we suspect that triggered star formation may be at work in M8 E.

In order to investigate the possibility of past triggered star formation in M8 E, we examined the population of YSOs in this region. Dewangan \& Anandarao (2010) cataloged class 0/I and class I YSOs using Spitzer/IRAC four-channel photometry. The YSOs were classified based on their evolutionary sequence, 


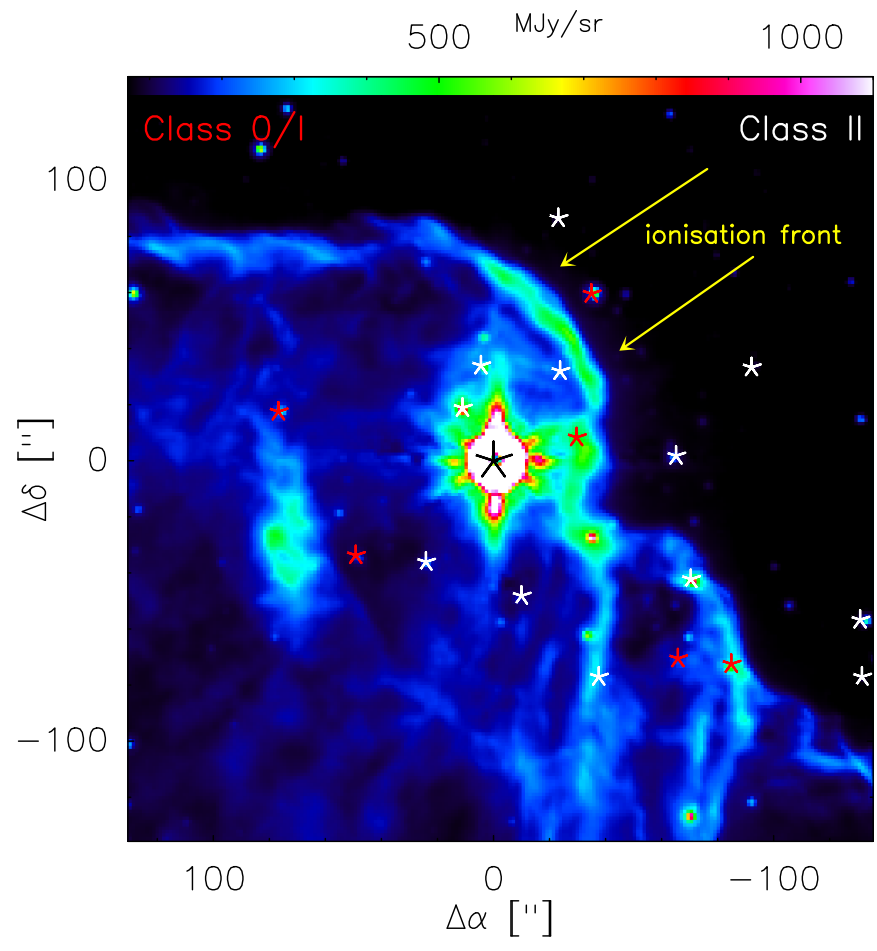

Fig. 10. GLIMPSE $8 \mu \mathrm{m}$ PAH emission distribution is shown in color scale along with the class 0/I (red) and class II (white) population of YSOs in M8 E. The IF, the bright rimmed structure, indicated by arrows (yellow). Position offsets are relative to the position of M8E-IR (marked with an asterisk) given in Sect. 2.

Lada (1991) introduced the nomenclature of class I-III sources: class I sources are the youngest, most deeply embedded, whereas class II sources are Classical T Tau stars still surrounded by circumstellar material, and finally, class III objects that are "naked" $\mathrm{T}$ Tau stars that have shed their envelopes. A still earlier phase than class I was reported by Andre et al. (1993). A total of 27 classical T Tauri stars (CTTs), which are per definitionem class II YSOs, have been identified in the region from multi-observatory IR data by Arias et al. (2006, 2007). We list the YSOs that lie close to M8 E in Table B.1, and Fig. 10 shows their distribution on the GLIMPSE $8 \mu \mathrm{m}$ emission image. It can be seen that the younger YSOs, class 0/I, predominantly appear to be associated with the dense gas swept up by the IF (east of M8 E), while class II YSOs (from the older generation) are distributed evenly at either side of the IF.

We note that in their recent study of NGC 6530, conducted in the framework of the Gaia-ESO survey, Prisinzano et al. (2019) also interpreted the distribution of classical T Tauri CTTs relative to the open cluster's O-type stars as evidence for triggered star formation in M8 E. This revised the view of Kalari et al. (2015), who in their earlier work also studying CTTs in NGC 6530 and saw no such evidence.

In order to estimate the velocity of the IF, we assumed that it is moving at a constant speed. The relative distance between the centers of the class 0/I and class II distributions of YSOs is about $0.5 \mathrm{pc}$. We assumed a typical upper limit of $\sim 2 \mathrm{Myr}$ for the age of class II YSOs. For the open cluster NGC 6530, Damiani et al. (2019) used Gaia data to find sequential star formation over an age range from 0.5 to $5 \mathrm{Myr}$; here we adopted $2 \mathrm{Myr}$. For the age of Class 0/I YSOs we took $\sim 0.27 \mathrm{Myr}$, the average of the ages of Class $0(\sim 0.1 \mathrm{Myr})$ and Class I ( $\sim 0.44 \mathrm{Myr})$ YSOs (Evans et al. 2009). With the ages, we determine a lower limit on the speed of the IF $\sim 0.26 \mathrm{~km} \mathrm{~s}^{-1}$, which is in accordance with the maximum predicted IF speed range of $\sim 0.5-1 \mathrm{~km} \mathrm{~s}^{-1}$ in PDR-type environments such as that of the Orion Bar (Störzer \& Hollenbach 1998).

\section{Conclusions}

We reported observations of diffuse and dense molecular gas tracers toward M8 E in the $3 \mathrm{~mm}$ regime using the EMIR receiver of the IRAM $30 \mathrm{~m}$ telescope. For the first time, we presented velocity-integrated intensity maps of the $J=1 \rightarrow 0$ transition of a selection of molecules in this region: ${ }^{12} \mathrm{CO},{ }^{13} \mathrm{CO}, \mathrm{N}_{2} \mathrm{H}^{+}$, $\mathrm{HCN}, \mathrm{H}^{13} \mathrm{CN}, \mathrm{HCO}^{+}, \mathrm{H}^{13} \mathrm{CO}^{+}, \mathrm{HNC}$, and $\mathrm{HN}^{13} \mathrm{C}$ in this region.

From ancillary data, analyzed in Sect. 3.1, we found that the $870 \mu \mathrm{m}$ submillimeter dust continuum emission probes the dense gas that causes star formation in M8 E, while the 8 and $22 \mu \mathrm{m}$ emission traces the warm gas. In Sect. 4, using several LTE and non-LTE methods, we determined the warm and cool gas temperatures $\sim 80$ and $30 \mathrm{~K}$, respectively, and the $\mathrm{H}_{2}$ volume densities in the range of $10^{4}$ to $10^{6} \mathrm{~cm}^{-3}$. Toward M8 E, we employed the molecular cloud thermometer $\mathrm{CH}_{3} \mathrm{CCH}$ to determine the temperature of the dense gas component. We summarized the differences in the estimated abundances of various species observed toward M8 E and M8-Main in Sect. 5. Star formation is probably occurring in a denser environment in M8 E than in M8-Main, placing M8 $\mathrm{E}$ at an earlier stage of star formation. Furthermore, the emission from the $J=1 \rightarrow 0$ transition of $\mathrm{N}_{2} \mathrm{H}^{+}$and $\mathrm{HN}^{13} \mathrm{C}$ is spatially most concentrated and is consistent with dense and cool gas (as probed by $\mathrm{CH}_{3} \mathrm{CCH}$ ).

From the geometry of the region and the distribution of the YSOs in M8 E, we find that large quantity of gas is being compressed and swept away by the IF of the expanding central H II region of M8. Based on the ages of different classes of the YSO population found in M8 E, we estimate that the IF is moving at least at a speed of $\sim 0.26 \mathrm{~km} \mathrm{~s}^{-1}$. We therefore conclude that an earlier generation of stars in the cluster NGC 6530 is responsible for the triggered star formation we observe in M8 E.

\section{Outlook}

Compared to the power house $\mathrm{O}$ stars that excite the bulk of M8 (see Sect. 1), the YSOs embedded in M8 E, with spectral types equivalent to B0 and B2, together with a few sources of still lower luminosity, clearly have a much weaker effect on the region, now and in the future. Nevertheless, as shown here, studies of M8 and its environs, with M8 E the so far best-studied example, allow interesting studies of the outcome of recent (M8-Main) and ongoing star formation that is triggered by the former.

The purpose of this paper was to map out the extent of the molecular material in M8 E and to derive its basic properties by analyzing data from ground-state transitions of common molecules. Higher- $J$ lines of these and other molecules measured with the APEX telescope will allow a comprehensive characterization of the energetics, density, and chemistry of the molecular gas, including the powerful outflow. In particular, the chemical richness of the source will be explored by data obtained in deep integrations toward M8E-IR, which for the $3 \mathrm{~mm}$ band are already in hand.

Much of the gaseous content of the wider M8 region remains unexplored, along with embedded YSOs that it may harbor, and warrants further exploration: in the $450 \mu \mathrm{m}$ continuum image (Fig. 1), many other dust condensations are clearly visible whose 
star-forming activity has not yet been investigated, for example, those in the striking $\sim 5 \mathrm{pc}$ long curved string with M8 $\mathrm{E}$ at its eastern end and the $\sim 1 \mathrm{pc}$ long north-south ridge abutting NGC 6530. Future systematic studies of the M8 region might discover objects comparable to M8 E, if less luminous, and in yet earlier stages of star formation. These objects hold the promise of finding more evidence for triggered star formation.

Acknowledgements. M. Tiwari was supported for this research by the International Max Planck Research School (IMPRS) for Astronomy and Astrophysics at the Universities of Bonn and Cologne. We thank Nina Brinkmann for helpful discussions.

\section{References}

Anderson, L. D., Bania, T. M., Balser, D. S., et al. 2014, ApJS, 212, 1 Andre, P., Ward-Thompson, D., \& Barsony, M. 1993, ApJ, 406, 122

Arias, J. I., Barbá, R. H., Maíz Apellániz, J., Morrell, N. I., \& Rubio, M. 2006, MNRAS, 366, 739

Arias, J. I., Barbá, R. H., \& Morrell, N. I. 2007, MNRAS, 374, 1253

Arias, J. I., Barbá, R. H., Gamen, R. C., et al. 2010, ApJ, 710, L30

Bania, T. M., Anderson, L. D., Balser, D. S., \& Rood, R. T. 2010, ApJ, 718, L106

Barnes, A. T., Kauffmann, J., Bigiel, F., et al. 2020, MNRAS, 497, 1972

Benjamin, R. A., Churchwell, E., Babler, B. L., et al. 2003, PASP, 115, 953

Bergin, E. A., Goldsmith, P. F., Snell, R. L., \& Ungerechts, H. 1994, ApJ, 431, 674

Brinkmann, N., Wyrowski, F., Kauffmann, J., et al. 2020, A\&A, 636, A39

Carey, S. J., Noriega-Crespo, A., Mizuno, D. R., et al. 2009, PASP, 121, 76

Carter, M., Lazareff, B., Maier, D., et al. 2012, A\&A, 538, A89

Churchwell, E., Babler, B. L., Meade, M. R., et al. 2009, PASP, 121, 213

Dame, T. M., Hartmann, D., \& Thaddeus, P. 2001, ApJ, 547, 792

Damiani, F., Flaccomio, E., Micela, G., et al. 2004, ApJ, 608, 78

Damiani, F., Prisinzano, L., Micela, G., \& Sciortino, S. 2019, A\&A, 623, A25

Daniel, F., Dubernet, M. L., Meuwly, M., Cernicharo, J., \& Pagani, L. 2005, MNRAS, 363, 1083

Dewangan, L. K., \& Anandarao, B. G. 2010, ArXiv e-prints, [arXiv:1005 . 1148]

Draine, B. T. 2011, Physics of the Interstellar and Intergalactic Medium (Princeton: Princeton University Press)

Dumouchel, F., Faure, A., \& Lique, F. 2010, MNRAS, 406, 2488

Elmegreen, B. G., \& Lada, C. J. 1977, ApJ, 214, 725

Evans, N. J., I., Dunham, M. M., Jørgensen, J. K., et al. 2009, ApJS, 181, 321

Flower, D. R. 1999, MNRAS, 305, 651

Giannetti, A., Brand, J., Massi, F., Tieftrunk, A., \& Beltrán, M. T. 2012, A\&A, 538, A41

Giannetti, A., Leurini, S., Wyrowski, F., et al. 2017, A\&A, 603, A33

Goldsmith, P. F., \& Kauffmann, J. 2017, ApJ, 841, 25

Güsten, R., Booth, R. S., Cesarsky, C., et al. 2006, Proc. SPIE, 6267, 626714

Hernández Vera, M., Lique, F., Dumouchel, F., Hily-Blant, P., \& Faure, A. 2017, MNRAS, 468, 1084

Hollenbach, D. J., \& Tielens, A. G. G. M. 1999, Rev. Mod. Phys., 71, 173

Hunter, J. D. 2007, Comput. Sci. Eng., 9, 90
Jaynes, E. T. 2003, Probability Theory: The Logic of Science, ed. G. L. Bretthorst (Cambridge, UK: Cambridge University Press)

Kalari, V. M., Vink, J. S., Drew, J. E., et al. 2015, MNRAS, 453, 1026

Kauffmann, J., Bertoldi, F., Bourke, T. L., Evans, N. J., I., \& Lee, C. W. 2008 A\&A, 487, 993

Kauffmann, J., Goldsmith, P. F., Melnick, G., et al. 2017, A\&A, 605, L5

Kim, C.-G., Ostriker, E. C., \& Kim, W.-T. 2013, ApJ, 776, 1

Lada, C. J. 1991, NATO Advanced Science Institutes (ASI) Series C, 342, 329

Lada, C. J., Gottlieb, C. A., Gottlieb, E. W., \& Gull, T. R. 1976, ApJ, 203, 159

Linz, H., Stecklum, B., Follert, R., et al. 2008, J. Phys. Conf. Ser., 131, 012024

Mangum, J. G., \& Shirley, Y. L. 2015, PASP, 127, 266

Maret, S., Hily-Blant, P., Pety, J., Bardeau, S., \& Reynier, E. 2011, A\&A, 526, A47

Milam, S. N., Savage, C., Brewster, M. A., Ziurys, L. M., \& Wyckoff, S. 2005, ApJ, 634, 1126

Mitchell, G. F., Hasegawa, T. I., \& Schella, J. 1992, ApJ, 386, 604

Molinari, S., Brand, J., Cesaroni, R., \& Palla, F. 1996, A\&A, 308, 573

Nakamura, F., Oyamada, S., Okumura, S., et al. 2019, PASJ, 32

Patil, A., Huard, D., \& Fonnesbeck, C. 2010, J. Stat. Softw. Art., 35, 1

Peng, T.-C., Wyrowski, F., Zapata, L. A., Güsten, R., \& Menten, K. M. 2012, A\&A, 538, A12

Pety, J. 2005, SF2A-2005: Semaine de l'Astrophysique Francaise, eds. F. Casoli, T. Contini, J. M. Hameury, \& L. Pagani, 721

Pety, J., Guzmán, V. V., Orkisz, J. H., et al. 2017, A\&A, 599, A98

Prisinzano, L., Damiani, F., Micela, G., \& Sciortino, S. 2005, A\&A, 430, 941

Prisinzano, L., Damiani, F., Kalari, V., et al. 2019, A\&A, 623, A159

Rauw, G., Sana, H., Spano, M., et al. 2012, A\&A, 542, A95

Reid, M. J., Menten, K. M., Brunthaler, A., et al. 2014, ApJ, 783, 130

Schöier, F. L., van der Tak, F. F. S., van Dishoeck, E. F., \& Black, J. H. 2005 A\&A, 432, 369

Schuller, F., Menten, K. M., Contreras, Y., et al. 2009, A\&A, 504, 415

Shirley, Y. L. 2015, PASP, 127, 299

Snyder, L. E., \& Buhl, D. 1973, Nat. Phys. Sci., 243, 45

Störzer, H., \& Hollenbach, D. 1998, ApJ, 495, 853

Thiel, V., Belloche, A., Menten, K. M., et al. 2019, A\&A, 623, A68

Tielens, A. G. G. M. 2008, ARA\&A, 46, 289

Tielens, A. G. G. M. 2010, The Physics and Chemistry of the Interstellar Medium (Cambridge: Cambridge University Press)

Tielens, A. G. G. M. 2013, Rev. Mod. Phys., 85, 1021

Tiwari, M., Menten, K. M., Wyrowski, F., et al. 2018, A\&A, 615, A158

Tiwari, M., Menten, K. M., Wyrowski, F., et al. 2019, A\&A, 626, A28

Tothill, N. F. H., White, G. J., Matthews, H. E., et al. 2002, ApJ, 580, 285

Tothill, N. F. H., Gagné, M., Stecklum, B., \& Kenworthy, M. A. 2008, Handbook of Star Forming Regions, ed. B. Reipurth (San Francisco: ASP), 533

Urquhart, J. S., Thompson, M. A., Morgan, L. K., et al. 2007, A\&A, 467, 1125

van der Tak, F. F. S., Black, J. H., Schöier, F. L., Jansen, D. J., \& van Dishoeck, E. F. 2007, A\&A, 468, 627

Watanabe, Y., Nishimura, Y., Harada, N., et al. 2017, ApJ, 845, 116

White, G. J., Tothill, N. F. H., Matthews, H. E., et al. 1997, A\&A, 323, 529

Woodward, C. E., Pipher, J. L., Helfer, H. L., et al. 1986, AJ, 91, 870

Wright, E. L., Eisenhardt, P. R. M., Mainzer, A. K., et al. 2010, AJ, 140, 1868

Yang, B., Stancil, P. C., Balakrishnan, N., \& Forrey, R. C. 2010, ApJ, 718, 1062

Young, E. T., Becklin, E. E., Marcum, P. M., et al. 2012, ApJ, 749, L17

Zhang, Q., Hunter, T. R., Brand, J., et al. 2005, ApJ, 625, 864 


\section{Appendix A: Spatial distribution of the $26 \mathrm{~km} \mathrm{~s}^{-1}$ molecular cloud}
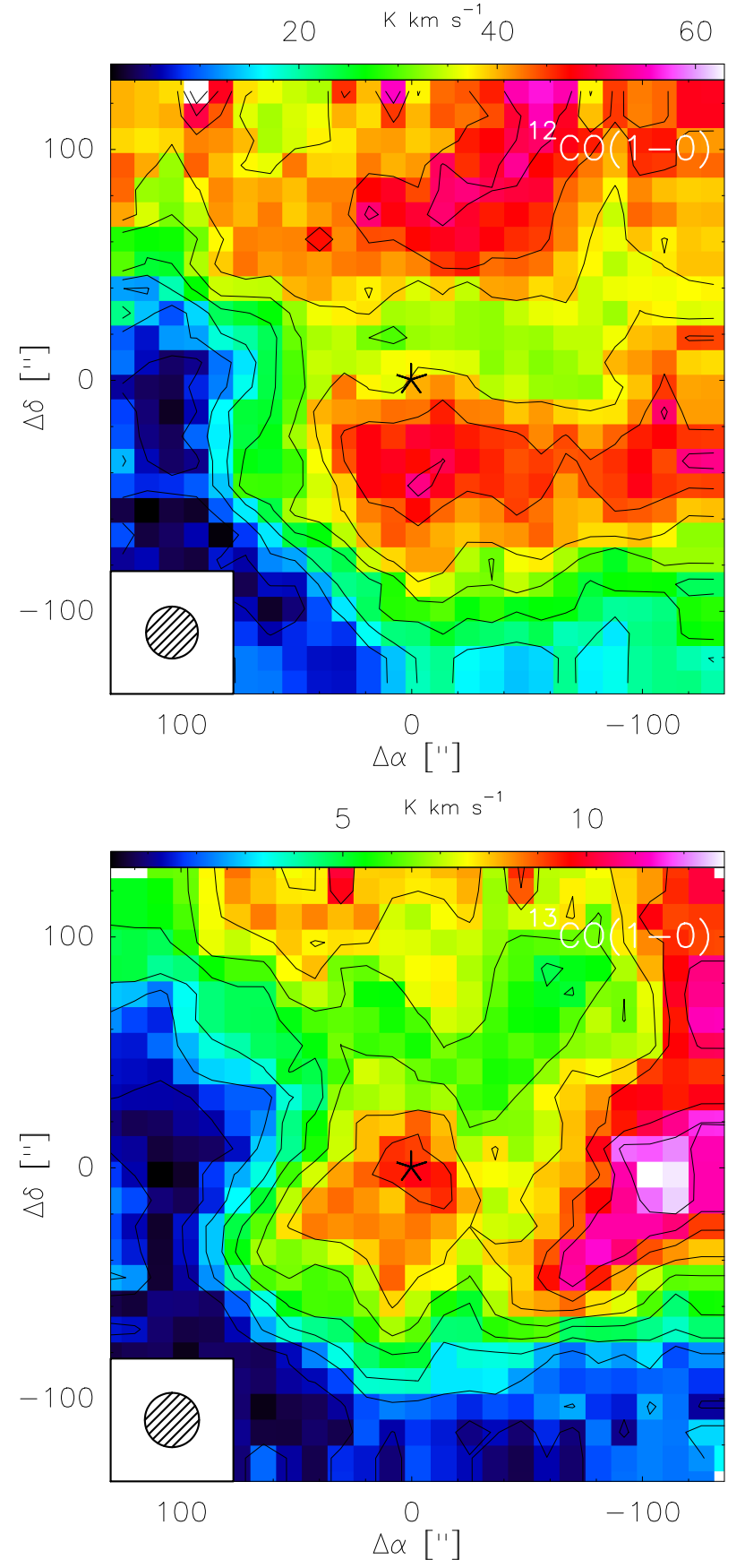

Fig. A.1. Intensity color maps of the $J=1 \rightarrow 0$ transition of ${ }^{12} \mathrm{CO}$ and ${ }^{13} \mathrm{CO}$ integrated within the velocity range of $22-30 \mathrm{~km} \mathrm{~s}^{-1}$. Position offsets are relative to the position of M8E-IR (marked with an asterisk) given in Sect. 2.

Velocity-integrated intensity maps of $J=1 \rightarrow 0$ transitions of ${ }^{12} \mathrm{CO}$ and ${ }^{13} \mathrm{CO}$ for the molecular cloud with $v_{\mathrm{LSR}}=26 \mathrm{~km} \mathrm{~s}^{-1}$ are shown in Fig. A.1. Similar to the main molecular cloud of M8 E (at $v_{\mathrm{LSR}}=11 \mathrm{~km} \mathrm{~s}^{-1}$ ), which is associated with M8-Main, the emission from the $+26 \mathrm{~km} \mathrm{~s}^{-1}$ molecular cloud has a local maximum toward M8E-IR. Unlike the main molecular cloud whose emission is abruptly stops southwest of M8E-IR because it is affected by M8 Main, however, the emission distribution of this cloud, which is located along the LOS to M8, has a much more widespread distribution.

Using the revised kinematic distance calculator accessible on the website of the Bar and Spiral Structure Legacy (BeSSeL) Survey $^{7}$ that uses values for the Galactic parameters published by Reid et al. (2014), we calculate for $v_{\mathrm{LSR}}=26 \mathrm{~km} \mathrm{~s}^{-1}$ near and far heliocentric kinematic distances of $4.08_{-0.73}^{+0.55}$ and $12.05_{-0.45}^{+0.58} \mathrm{kpc}$, respectively, and for $v_{\mathrm{LSR}}=11 \mathrm{~km} \mathrm{~s}^{-1}$ we find a (near) distance of $2.15_{-1.62}^{+1.07} \mathrm{kpc}$, which is consistent with the parallax distance for M8 $(1.35 \mathrm{kpc})$.

\section{Appendix B: YSO population in M8 E}

Table B.1. Identified YSOs in M8 E as reported in Dewangan \& Anandarao (2010).

\begin{tabular}{ccc}
\hline \hline Right Ascension (J2000) & Declination (J200) & Object $^{(a)}$ \\
\hline \multicolumn{3}{c}{ Class $0 / \mathrm{I}$} \\
\hline 18:04:47.09 & $-24: 27: 55.4$ & 58 \\
$18: 04: 48.42$ & $-24: 27: 53.8$ & 59 \\
$18: 04: 50.37$ & $-24: 14: 25.5$ & 60 \\
$18: 04: 50.62$ & $-24: 25: 42.2$ & 61 \\
$18: 04: 56.77$ & $-24: 27: 16.4$ & 63 \\
$18: 04: 58.82$ & $-24: 26: 24.1$ & 64 \\
\hline \multicolumn{3}{c}{ Class II } \\
$18: 04: 43.53$ & $-24: 27: 38.7$ & 152 \\
$18: 04: 43.65$ & $-24: 27: 59.1$ & 153 \\
$18: 04: 46.41$ & $-24: 26: 08.0$ & 156 \\
$18: 04: 48.05$ & $-24: 27: 24.6$ & 157 \\
$18: 04: 48.56$ & $-24: 26: 40.7$ & 158 \\
$18: 04: 50.23$ & $-24: 27: 59.4$ & 159 \\
$18: 04: 51.53$ & $-24: 24: 17.4$ & 160 \\
$18: 04: 51.57$ & $-24: 26: 10.9$ & 161 \\
$18: 04: 52.64$ & $-24: 27: 30.8$ & 163 \\
$18: 04: 53.48$ & $-24: 26: 08.7$ & 164 \\
\hline
\end{tabular}

Notes. ${ }^{(a)}$ Refers to the YSO number as mentioned in the catalog reported by Dewangan \& Anandarao (2010). 